KODAI MATH. SEM. REP.

19 (1967), 257-288

\title{
DIFFERENTIAL GEOMETRY OF FIBRED SPACES
}

\author{
By Kentaro Yano and Shigeru Ishihara
}

\section{Introduction.}

To obtain a unified field theory of gravitation and electromagnetism, Kaluza [4] ${ }^{1)}$ and Klein [5] used a five dimensional Riemannian manifold admitting an infinitesimal translation and identified a point in the space-time with a trajectory of the translation in this five dimensional Riemannian manifold.

On the other hand, to obtain a representation of an $n$-dimensional manifold with projective connection, van Dantzig [2], Haantjes [3], Schouten [7], Thomas [8], Veblen [9], Whitehead [11], one of the present authors [12], [13], [16] and others used an $(n+1)$-dimensional manifold with affine connection admitting a vector field which is concurrent and which defines an infinitesimal affine transformation, and they identified a point in the manifold with projective connection with a trajectory of the infinitesimal affine transformation in the manifold with affine connection. To study the manifold representing a manifold with projective connection, geometers of Dutch School used the so-called homogeneous curvilinear coordinates and those of Princeton School used the non-homogeneous curvilinear coordinates.

We call now fibred spaces such manifolds appearing in the unified field theory and in the theory of representation of projective space. The fibred spaces have been studied by Davies [14], Mutō [6] and the present authors [15].

The purpose of the present paper is to develop systematically the theory of fibred spaces. In $\S 1$ we define the fibred space and discuss tensor fields, invariant affine connection, induced affine connection and van der Waerden-Bortolotti covariant differentiation in the fibred space. We develop in $\$ 2$ the tensor calculus in fibred spaces in terms of local coordinates. $\S 3$ is devoted to the study of some special cases in which the so-called structure field satisfies certain conditions. We study the structure equations in $\S 4$ and the fibring in the flat space in $\S 5$. In the last $\S 6$, we examine manifolds with projective connection from our point of view.

\section{CONTENT}

§1. Fibred spaces.

$\S 2$. The tensor calculus in fibred spaces.

$\S 3$. Special cases.

$\S 4$. Structure equations.

Received January 12, 1967.

1) The number between brackets refers to the Reference at the end of the paper. 
$\S 5$. The fibring in flat spaces.

§6. Manifolds with projective connection.

\section{$\S 1$. Fibred spaces.}

Let $\tilde{M}$ and $M$ be two differentiable manifolds ${ }^{2)}$ of dimension $n+1$ and $n$ respectively, and assume that there exists a differentiable mapping $\pi: \tilde{M} \rightarrow M$, which is onto and of the maximum rank $n$. Then the inverse image $\pi^{-1}(\mathrm{P})$ of a point $\mathrm{P}$ of $M$ is a 1 -dimensional submanifold of $\tilde{M}$. Denoting $\pi^{-1}(\mathrm{P})$ by $F_{\mathrm{P}}$, we call the submanifold $F_{\mathrm{P}}$ the fibre over a point $\mathrm{P}$ of $M$. We assume in this paper that the fibre $F_{\mathrm{P}}$ is connected for every point $\mathrm{P}$ of $M$.

We assume that there are given in $\tilde{M}$ a vector field $\tilde{C}$ and a 1 -form $\tilde{\eta}$, the vector field $\widetilde{C}$ being non-zero and tangent to each fibre everywhere and the 1 -form $\tilde{\eta}$ satisfying the conditions

$$
\tilde{\eta}(\tilde{C})=1, \quad \mathcal{L} \tilde{\eta}=0,
$$

where the operator $\mathcal{L}$ denotes the Lie derivation with respect to $\tilde{C}$. The set $(\tilde{M}, M, \pi ; \tilde{C}, \tilde{\eta})$ satisfying these conditions is called a fibred space. We call $\tilde{M}$ and $M$ the total space and the base space of the fibred space respectively. The vector field $\tilde{C}$ and the 1 -form $\tilde{\eta}$ are called the structure field and the structure 1-form of the fibred space respectively. The $n$-dimensional distribution defined in $\tilde{M}$ by the equation $\tilde{\eta}=0$ is called the field of horizontal planes and the value of this distribution at each point is called the horizontal plane at that point. The mapping $\pi: \tilde{M} \rightarrow M$ is called the projection of the fibred space. If there is no fear of confusion, we call, for the sake of simplicity, a fibred space $(\tilde{M}, M, \pi ; \tilde{C}, \tilde{\eta})$ simply a fibred space $\tilde{M}$.

Tensor fields in fibred spaces. We shall here introduce some notations as follows:

1. $T(\tilde{M})$ is the tangent bundle of $\tilde{M}$.

2. $\mathscr{I}_{s}^{r}(\tilde{M})$ is the space of all tensor fields of type $(r, s)$, i.e., of contravariant degree $r$ and covariant degree $s$, in $\tilde{M}$.

3.

$$
\mathscr{I}(\tilde{M})=\sum_{r, s} \mathscr{I}_{s}^{r}(\tilde{M})
$$

The notations $T(M), \mathscr{I}_{s}^{r}(M)$ and $\mathscr{I}(M)$ denote the spaces corresponding to $T(\tilde{M})$, $\mathscr{I}_{s}^{r}(\tilde{M})$ and $\mathscr{I}(\tilde{M})$, respectively, with respect to $M$.

We shall introduce a linear endomorphism $T \rightarrow T^{H}$ of $\mathscr{I}(\tilde{M})$ which is characterized by the following properties:

$$
\tilde{f}^{H}=\tilde{f} \quad \text { for } \quad \tilde{f} \in \mathscr{I}_{0}^{\circ}(\tilde{M}) .
$$

2) The differentrability of the manifolds and of the objects we discuss is supposed always to be of $C^{\infty}$. 


$$
\begin{array}{lll}
\text { (H. 2) } & \tilde{X}^{H}=\tilde{X}-\tilde{\eta}(\tilde{X}) \tilde{C} & \text { for } \quad \tilde{X} \in \mathscr{I}_{0}^{1}(\tilde{M}) . \\
\text { (H. 3) } & \tilde{\omega}^{H}=\tilde{\omega}-\tilde{\omega}(\tilde{C}) \tilde{\eta} & \text { for } \quad \tilde{\omega} \in \mathscr{I}_{1}^{0}(\tilde{M}) .
\end{array}
$$$$
\text { (H. 4) } \quad(\tilde{S} \otimes \tilde{T})^{H}=\left(\tilde{S}^{H}\right) \otimes\left(\tilde{T}^{H}\right) \quad \text { for } \quad \tilde{S}, \tilde{T} \in \mathscr{T}(\tilde{M}) \text {. }
$$

The tensor field $\widetilde{T}^{H}$ is called the horizontal part of $\widetilde{T}$ for any $\tilde{T} \in \mathscr{T}(\tilde{M})$. If $\tilde{T}=\widetilde{T}^{H}$, $\widetilde{T}$ is said to be horizontal. On putting

$$
\tilde{T}^{V}=\tilde{T}-\widetilde{T}^{H} \quad \text { for } \quad \tilde{T} \in \mathscr{T}(\tilde{M})
$$

we call $\tilde{T}^{v}$ the non-horizontal part of $\tilde{T}$. Especially, for any elements $\tilde{X} \in \mathscr{I}_{0}^{1}(\tilde{M})$ and $\tilde{\omega} \in \mathscr{I}_{1}^{0}(\tilde{M}), \tilde{X}^{V}$ and $\tilde{\boldsymbol{\omega}}^{V}$ are called the vertical parts of $\tilde{X}$ and $\tilde{\boldsymbol{\omega}}$ respectively. If $\tilde{X}=\tilde{X}^{v}, \tilde{\omega}=\tilde{\omega}^{V}$, they are said to be vertical. We shall now introduce notations as follows:

4. $\mathscr{I}^{H}(\tilde{M})$ is the space of all horizontal tensor fields in $\tilde{M}$. $\mathscr{I}^{H}(\tilde{M}) \subset \mathscr{I}(\tilde{M})$.

A tensor field $\tilde{T}$ in $\tilde{M}$ is said to be invariant if $\mathcal{L} \widetilde{T}=0$ holds. We shall here introduce some notations as follows:

5. $\mathcal{g}(\tilde{M})$ is the space of all invariant tensor fields in $\tilde{M} . \quad \mathcal{I}(\tilde{M}) \subset \mathscr{I}(\tilde{M})$.

6.

$$
\mathcal{G}_{s}^{r}(\tilde{M})=\mathcal{G}(\tilde{M}) \cap \mathscr{I}_{s}^{r}(\tilde{M}) \text {. }
$$

7.

$$
\mathscr{I}^{H}(\tilde{M})=\mathcal{G}(\tilde{M}) \cap \mathscr{I}^{H}(\tilde{M}), \quad \mathcal{G}_{s}^{r}(\tilde{M})=\mathcal{I}^{H}(\tilde{M}) \cap \mathscr{I}_{s}^{r}(\tilde{M})
$$

Thus we have

$$
\mathcal{I}(\tilde{M})=\sum_{r, s} \mathcal{O}_{s}^{r}(\tilde{M}), \quad \mathcal{I}^{H}(\tilde{M})=\sum_{r, s} \mathcal{S}_{s}^{r}(\tilde{M}) .
$$

8. $\mathcal{G}(\tilde{M}) \# \mathcal{G}^{H}(\tilde{M})$ denotes the formal tensor product, i.e., the tensor product of $\mathcal{I}(\tilde{M})$ and $\mathcal{G}^{H}(\tilde{M})$ regarded as two abstract tensor spaces over $\tilde{M}$.

We denote by $j: \mathcal{I}^{H}(\tilde{M}) \rightarrow \mathcal{G}(\tilde{M})$ the natural injection. We shall now introduce a linear homomorphism $i: \mathcal{I}(\tilde{M}) \# \mathcal{G}^{H}(\tilde{M}) \rightarrow \mathcal{G}(\tilde{M})$ which is characterized by the following properties:

$$
\begin{aligned}
& i(\stackrel{*}{T})=j(\stackrel{*}{T}) \in \mathcal{G}(\tilde{M}) \quad \text { for } \quad \stackrel{*}{T} \in \mathcal{I}^{H}(\tilde{M}) . \\
& i(\widetilde{T})=\tilde{T} \quad \text { for } \quad \tilde{T} \in \mathcal{G}(\tilde{M}) \text {. } \\
& i(\tilde{S} \# \stackrel{*}{T})=i(\tilde{S}) \otimes i(\stackrel{*}{T}) \quad \text { for } \quad \tilde{S} \in \mathcal{G}(\tilde{M}), \quad \stackrel{*}{T} \in \mathcal{I}^{H}(\tilde{M}) .
\end{aligned}
$$

We shall introduce a linear homomorphism $T \rightarrow T^{L}$ of $\mathscr{I}(M)$ onto $\mathcal{G}^{H}(\tilde{M})$, which is characterized by the following properties:

$$
f^{L}=f \circ \pi \quad \text { for } f \in \mathscr{I}_{0}^{\circ}(M),
$$

(L. 2) for any element $X \in \mathscr{I}_{0}^{1}(M)$ there exists a unique element $X^{L} \in \mathcal{G}^{H}(\tilde{M})$ such that 


$$
\pi X^{L}=X
$$

$$
\omega^{L}=* \pi \omega \quad \text { for } \quad \omega \in \mathscr{I}_{1}^{0}(M)
$$

$$
(S \otimes T)^{L}=\left(S^{L}\right) \otimes\left(T^{L}\right) \quad \text { for } \quad S, T \in \mathscr{I}(M),
$$

where the differential mapping of the projection $\pi: \tilde{M} \rightarrow M$ is denoted also by $\pi$ and the dual mapping of the differential mapping $\pi$ is denoted by $* \pi$. For an element $T \in \mathscr{I}(M), T^{L}$ is called its lift.

We shall here introduce a linear homomorphism $p: \mathcal{G}(\tilde{M}) \rightarrow \mathscr{I}(M)$ which is characterized by the following properties:

$$
(p \tilde{f})(\mathrm{P})=\tilde{f}(\tilde{\mathrm{P}}) \quad \text { for } \quad \tilde{f} \in \mathcal{I}_{0}^{0}(\tilde{M}),
$$

where $\widetilde{\mathrm{P}}$ is an arbitrary point of $F_{\mathrm{P}}, \mathrm{P}$ being an arbitrary point of $M$.

$$
(p \tilde{X}) f=p\left(\tilde{X} f^{L}\right) \quad \text { for } \quad \tilde{X} \in \mathcal{I}_{0}^{1}(\tilde{M}),
$$

$f$ being an arbitrary function in $M$.

$$
(p \tilde{\omega})(X)=p\left(\tilde{\omega}\left(X^{L}\right)\right) \quad \text { for } \quad \tilde{\omega} \in \mathcal{I}_{1}^{0}(\tilde{M})
$$

$X$ being an arbitrary vector field in $M$.

$$
p(\tilde{S} \otimes \tilde{T})=(p \tilde{S}) \otimes(p \tilde{T}) \quad \text { for } \tilde{S}, \tilde{T} \in \mathcal{G}(\tilde{M}) .
$$

For an element $\tilde{T}$ of $\mathcal{G}(\tilde{M}), p \tilde{T}$ is called its projection.

Taking account of the definition above, we can easily verify the following formulas:

$$
p \widetilde{T}=p\left(\tilde{T}^{H}\right), \quad p\left(\tilde{T}^{v}\right)=0 \quad \text { for } \quad \widetilde{T} \in \mathcal{I}(\tilde{M})
$$

$$
p\left(T^{L}\right)=T
$$

for $T \in \mathscr{I}(M)$.

$$
(p \tilde{T})^{L}=\tilde{T}^{H}
$$

for $\tilde{T} \in \mathcal{G}(\tilde{M})$.

$$
(p \tilde{f})^{L}=\tilde{f}
$$

for $\tilde{f} \in \mathcal{G}_{0}^{\circ}(\tilde{M})$.

Because of (1.4) and (1.5) we see that the projection $p: \mathcal{G}(\tilde{M}) \rightarrow \mathscr{I}(M)$ induces an isomorphism $p: \mathcal{G}^{H}(\tilde{M}) \rightarrow \mathscr{I}(M)$ and the operation taking lifts is the inverse of the restriction of the projection $p$ to $\mathcal{G}^{H}(\tilde{M})$.

We have now

Proposition 1. 1. For any two elements $\tilde{X}, \tilde{Y} \in \mathcal{I}_{0}^{1}(\tilde{M})$, we have 


$$
\begin{array}{rlrl}
{[\tilde{X}, \tilde{Y}] \in \mathcal{I}_{0}^{1}(\tilde{M}),} & {[\tilde{C}, \tilde{X}]} & =0, \\
{[\tilde{X}, \tilde{Y}]^{H}=\left[\tilde{X}^{H}, \tilde{Y}^{H}\right]^{H},} & {[\tilde{X}, \tilde{Y}]^{V}} & =\left[\tilde{X}^{H}, \tilde{Y}^{H}\right]^{V} \\
& =-2 \tilde{\Omega}(\tilde{X}, \tilde{Y}) \tilde{C},
\end{array}
$$

and

$$
p[\tilde{X}, \tilde{Y}]=[p \tilde{X}, p \tilde{Y}]
$$

where $\tilde{\Omega}$ is an invariant 2-form defined by

$$
\tilde{\Omega}=d \tilde{\eta} .
$$

For any two elements $X, Y \in \mathscr{I}_{0}^{1}(M)$, we have

$$
\left[X^{L}, Y^{L}\right]^{H}=[X, Y]^{L}, \quad\left[X^{L}, Y^{L}\right]^{V}=-2 \tilde{\Omega}\left(X^{L}, Y^{L}\right) \tilde{C}
$$

(cf. Yano and Ishihara [15]).

We now introduce in $M$ a 2 -form $\Omega$ such that

$$
\Omega=p \tilde{\Omega}=p(d \tilde{\eta})
$$

Thus we have

Proposition 1.2. For any element $\tilde{\omega} \in \mathcal{G}_{1}^{\circ}(\tilde{M})$, we have

$$
\begin{gathered}
d \tilde{\omega} \in \mathcal{I}_{2}^{0}(\tilde{M}), \\
p(d \tilde{\omega})=d(p \tilde{\omega})+p(\tilde{\omega}(\tilde{C})) \Omega .
\end{gathered}
$$

When $\tilde{\omega} \in \mathcal{G}_{1}^{H_{0}^{0}}(\tilde{M})$, we have

$$
p(d \tilde{\omega})=d(\tilde{\omega})
$$

(cf. Yano and Ishihara [15]).

Taking account of $d \tilde{\Omega}=0$, which is a direct consequence of (1.9), we have $d \Omega=0$ because of (1.10). Thus the 2 -form $\Omega$ is closed. We denote by [ $\Omega$ ] the element of $H_{2}(M)$ determined in $M$ by the closed 2-form $\Omega$, where $H_{2}(M)$ denotes the second cohomology group of the base space $M$.

If we omit the structure 1 -form $\tilde{\eta}$ from the set $(\tilde{M}, M, \pi ; \tilde{C}, \tilde{\eta})$, then we have a set $(\tilde{M}, M, \pi ; \widetilde{C})$ which is called a fibred space without horizontal planes. Suppose that, in a fibred space $(\tilde{M}, M, \pi ; \tilde{C})$ without horizontal planes, there are given two structure 1-forms $\tilde{\eta}$ and ' $\tilde{\eta}$, i.e., suppose that $\mathcal{L} \tilde{\eta}=\mathcal{L}^{\prime} \tilde{\eta}=0$ and $\tilde{\eta}(\tilde{C})={ }^{\prime} \tilde{\eta}(\tilde{C})=1$ hold. Then, putting $\zeta={ }^{\prime} \tilde{\eta}-\tilde{\eta}$, we find because of (1.11) 


$$
p\left(d^{\prime} \tilde{\eta}\right)=p(d \tilde{\eta})+d(p \zeta)
$$

which is equivalent to the condition

$$
' \Omega=\Omega+d(p \zeta)
$$

where $\Omega=p(d \tilde{\eta})$ and $' \Omega=p\left(d^{\prime} \tilde{\eta}\right)$. This equation shows that the two cohomology classes $[\Omega]$ and $\left[{ }^{\prime} \Omega\right]$ coincide with each other. Thus we have

Proposition 1.3. Let there be given two structure 1-forms $\tilde{\eta}$ and ' $\tilde{\eta}$ in a fibred space without horizontal planes. Then the two cohomology classes [Q] and ['S] coincide with each other, where $\Omega$ and ' $\Omega$ are determined in the base space $M$ by (1.11) in terms of $\tilde{\eta}$ and ' $\tilde{\eta}$, respectively. That is to say, to a fibred space without horizontal planes, there corresponds a unique cohomology class $[\Omega] \in H_{2}(M)$ in the base space $M$.

The unique cohomology class $[\Omega] \in H_{2}(M)$ corresponding to a fibred space $(\tilde{M}, M, \pi ; \tilde{C})$ without horizontal planes is called the characteristic class of the fibred space $(\tilde{M}, M, \pi ; \tilde{C})$ without horizontal planes.

Invariant affine connection in fibred space. Let there be given an affine connection $\tilde{V}$ in the total space $\tilde{M}$ such that $\tilde{V}$ is invariant under the infinitesimal transformation determined by the structure field $\tilde{C}$, i.e.,

$$
\mathcal{L}\left(\tilde{\nabla}_{\tilde{Y}} \tilde{X}\right)=\tilde{V}_{\tilde{Y}}(\mathcal{L} \tilde{X})+\tilde{V}_{[\tilde{C}, \tilde{Y}]} \tilde{X}
$$

for any vector fields $\tilde{X}$ and $\tilde{Y}$ in $\tilde{M}$. Such an affine connection $\tilde{\nabla}$ is called simply an invariant affine connection in $\tilde{M}$. When $\tilde{X}$ and $\tilde{Y}$ in (1.14) are invariant, this formula reduces to $\mathcal{L}\left(\tilde{\nabla}_{\tilde{Y}} \tilde{X}\right)=0$. Therefor $\tilde{\nabla}_{\tilde{Y}} \tilde{X}$ is an invariant vector field if $\tilde{X}$, $\tilde{Y}$ and $\tilde{\nabla}$ are invariant. Thus an invariant affine connection $\tilde{\nabla}$ operates in $\mathcal{G}(\tilde{M})$ as a derivation. Now, we shall define an affine connection $\nabla$ in the base space $M$ by the following property:

$$
\nabla_{Y} X=p\left(\tilde{\nabla}_{Y L} X^{L}\right)
$$

$X$ and $Y$ being arbitrary vector fields in $M$. The connection $\nabla$ thus defined is called the projection of the invariant affine connection $\tilde{\nabla}$ given in $\tilde{M}$ or the induced connection in $M$.

The torsion tensor $\tilde{T}$ of the affine connection $\tilde{\nabla}$ given in $\tilde{M}$ is by definition

$$
\tilde{T}(\tilde{X}, \tilde{Y})=\tilde{V} \tilde{X} \tilde{Y}-\tilde{V} \tilde{Y} \tilde{X}-[\tilde{X}, \tilde{Y}]
$$

$\tilde{X}$ and $\tilde{Y}$ being arbitrary vector fields in $\tilde{M}$. Taking account of the definition (1. 15) and Proposition 1.1, we see that the torsion tensor $\tilde{T}$ is invariant and its projection $T=p \tilde{T}$ coincides with the torsion tensor of the induced affine connection 
$\nabla$ in $M$. Thus we have

Proposition 1.4. Let $\tilde{\nabla}$ be an invariant affine connection in a fibred space $\tilde{M}$. When $\tilde{\nabla}$ is torsionless, so is also the affine connection $\nabla$ induced in $M$ from $\tilde{\nabla}$.

We now note the fact that the curvature tensor $\tilde{R}$ of an invariant affine connection $\tilde{\nabla}$ is also invariant. We shall study properties of the curvature tensor $\tilde{R}$ in $\S 4$.

We can prove easily the following formulas:

$$
p\left(\tilde{V}_{\widetilde{Y}} \widetilde{T}\right)=\nabla_{Y} T \quad \text { for } \quad \tilde{Y} \in \mathcal{G}^{H_{0}^{1}}(\tilde{M}), \quad \tilde{T} \in \mathcal{G}^{H}(\tilde{M}),
$$

where $Y=p \tilde{Y}$ and $T=p \tilde{T}$, and

$$
\nabla_{Y} T=p\left(\tilde{\nabla}_{Y L} T^{L}\right) \quad \text { for } \quad Y \in \mathscr{I}_{0}^{1}(M), \quad T \in \mathscr{I}(M),
$$

where $\tilde{\nabla}$ is an invariant affine connection in $\tilde{M}$ and $\nabla$ its projection.

Van der Waerden-Bortolotti covariant derivative. Suppose that an invariant affine connection $\tilde{\nabla}$ is given in the total space $\tilde{M}$ of a fibred space. Given an element $\tilde{Y} \in \mathcal{I}_{0}^{1}(\tilde{M})$, we define a derivation $\stackrel{*}{V}_{\tilde{Y}}$ in the formal tensor product $\mathcal{S}(\tilde{M}) \# \mathcal{G}^{H}(\tilde{M})$ by the following properties:

$$
\tilde{\nabla}_{\tilde{Y}}^{*} \tilde{T}=\tilde{\nabla}_{\tilde{Y}} \tilde{T} \quad \text { for } \quad \tilde{T} \in \mathcal{G}(\tilde{M}) \text {. }
$$

$$
\text { (W. 3) } \quad{ }^{*} \widetilde{Y}(\tilde{S} \# \stackrel{*}{T})=(\stackrel{*}{\nabla} \widetilde{Y} \widetilde{S}) \# \stackrel{*}{T}+\tilde{S} \#\left(\stackrel{*}{\nabla_{\tilde{Y}}} \stackrel{*}{T}\right) \quad \text { for } \quad \tilde{S} \in \mathcal{I}(\tilde{M}), \quad \stackrel{*}{T} \in \mathcal{I}^{H}(\tilde{M}) \text {. }
$$

For any element $\stackrel{*}{W}$ of $\mathcal{G}(\tilde{M}) \# \mathcal{G}^{H}(\tilde{M})$, the correspondence $\tilde{Y} \rightarrow \stackrel{*}{\rightarrow} \widetilde{Y} \tilde{W}$ defines an element $\stackrel{*}{\nabla} \stackrel{*}{W}$ of $\mathcal{G}(\tilde{M}) \# \mathcal{G}^{H}(\tilde{M}), \tilde{Y}$ being an arbitrary element of $\mathcal{G}_{0}^{1}(\tilde{M})$. We call $\stackrel{*}{\nabla} \stackrel{*}{W}$ the van der Waerden-Bortolotti covariant derivative of $\stackrel{*}{W}$.

REMARK. Let $G$ be a Lie group of 1 dimension, which is connected. Let there be given a principal fibre boundle $B=(\tilde{M}, M, G)$ with $G$ as its structure group, $\tilde{M}$ and $M$ being the total space and the base space respectively. Denote by $\tilde{C}$ the vector field in $\tilde{M}$ whose value at each point is the image of $C$ by the principal mapping, where $C$ is a non-zero element of the Lie algebra of $G$. Take an infinitesimal connection $\tilde{\eta}$ in $\tilde{M}$ and consider the set $(\tilde{M}, M, \pi ; \tilde{C}, \tilde{\eta}), \pi$ being the bundle projection of $B$. If we define in this case invariant tensor fields and invariant affine connections in $\tilde{M}$ as those which are invariant under the right-translation of the structure group $G$, then we can develop the same discussions as we had in this section. 


\section{$\S 2$. The tensor calculus in fibred spaces.}

Let $(\tilde{M}, M, \pi ; \tilde{C}, \tilde{\eta})$ be a fibred space. Since the projection $\pi$ is differentiable and of the maximum rank, there exists, for any point $\widetilde{\mathrm{P}}$ of $\tilde{M}$, a coordinate neighborhood $\tilde{U}$ of $\tilde{\mathrm{P}}$ such that $U=\pi(\tilde{U})$ is a coordinate neighborhood containing $\mathrm{P}=\pi(\tilde{\mathrm{P}})$ in $M$ and the intersection $\tilde{U} \cap F_{Q}$ is expressed by equations

$$
y^{1}=a^{1}, y^{2}=a^{2}, \cdots, y^{n}=a^{n},
$$

$a^{1}, a^{2}, \cdots$ and $a^{n}$ being constants, with respect to certain coordinates $\left(y^{1}, y^{2}, \cdots, y^{n+1}\right)$ defined in $\tilde{U}$, where $\mathrm{Q}$ is an arbitrary point of $U$. We call such a neighborhood $\tilde{U}$ a cylindrical neighborhood of $\tilde{M}$. We restrict ourselves only to cylindrical neighborhoods in $\tilde{M}$. Thus we call a cylindrical neighborhood of $\tilde{M}$ simply a neighborhood of $\tilde{M}$ in the sequel for the sake of simplicity.

Given a neighborhood $\tilde{U}$ of $\tilde{M}$, the set $(\tilde{U}, U, \pi ; \tilde{C}, \tilde{\eta})$ is a fibred space which is called the restriction of the given fibred space to $\tilde{U}$, where $U=\pi(\tilde{U}), \pi$ is the restriction of the projection $\pi: \tilde{M} \rightarrow M$, and $\widetilde{C}$ and $\tilde{\eta}$ are respectively the restriction of the structure field and the structure 1 -form in $\tilde{M}$. In the sequel, we shall identify the operations taking horizontal parts, lifts, projections and etc. in the fibred space $(\tilde{U}, U, \pi ; \tilde{C}, \tilde{\eta})$ with the corresponding operations in the given fibred space $(\tilde{M}, M, \pi ; \tilde{C}, \tilde{\eta})$ respectively.

Let there be given local coordinates $\left(x^{k}\right)=\left(x^{1}, x^{2}, \cdots, x^{n+1}\right)$ and $\left(\xi^{a}\right)=\left(\xi^{1}, \xi^{2}, \cdots, \xi^{n}\right)$ in a neighborhood $\tilde{U}$ of $\tilde{M}$ and in $U=\pi(\tilde{U})$ respectively. ${ }^{3)}$ We denote respectively by $E^{n}$ and $E_{\imath}$ the components of the structure field $\widetilde{C}$ and the structure 1 -form $\tilde{\eta}$ with respect to coordinates defined in $\left.\tilde{U} .^{4}\right)$ We may assume that, for an arbitrary point $\mathrm{P}$ with coordinates $\left(\xi^{a}\right)$ in $U, F_{\mathrm{P}} \cap \tilde{U}$ is represented by $n$ equations

$$
\xi^{a}=\xi^{a}\left(x^{h}\right)
$$

in $\tilde{U}$, where $n$ functions $\xi^{a}\left(x^{h}\right)$ are differentiable in $\tilde{U}$ and their Jacobian matrix $\left(\partial \xi^{a} / \partial x^{h}\right)$ is of rank $n$. We put

$$
E_{\imath}^{a}=\partial_{i} \xi^{a},
$$

where $\partial_{i}$ means the operator

$$
\partial_{i}=\frac{\partial}{\partial x^{2}}
$$

3) The indices $h, i, j, k, l, m$ run over the range $\{1,2, \cdots, n+1\}$ and the indices $a, b, c, d$, $e, f$ run over the range $\{1,2, \cdots, n\}$. The so-called Einstein's summation convention is used with respect to these two systems of indices.

4) Given a tensor field $\widetilde{T}$ in $\widetilde{M}$, its components with respect to local coordinates $\left(x^{h}\right)$ mean defined in $\widetilde{U}$, or, the components of $\widetilde{T}$ in $\widetilde{U}$ the components of $\widetilde{T}$ with respect to the natural frame $\left\{\partial / \partial x^{i}\right\}$ defined in $\widetilde{U}$. 
We then see that $n$ local covector fields $\zeta^{a}$ with components $E_{\imath}{ }^{a}$ are linearly independent in $\tilde{U}$. Since the structure field $\tilde{C}$ is tangent to fibres, we find

$$
E^{i} E_{\imath}=1, \quad E^{i} E_{\imath}^{a}=0,
$$

the first equaion being a direct consequence of $\tilde{\eta}(\tilde{C})=1$. The $n+1$ local covector fields $\zeta^{a}$ and $\tilde{\eta}$ are linearly independent and hence form a local field of coframes in $\tilde{U}$.

As a consequence of (2.3), we see that the inverse of the matrix $\left(E_{\imath}{ }^{a}, E_{i}\right)$ has the form

$$
\left(E_{\imath}^{a}, E_{i}\right)^{-1}=\left(\begin{array}{c}
E^{h} b \\
E^{h}
\end{array}\right)
$$

where, for each fixed index $b, E^{h}$ are components of a local vector field $\widetilde{B}_{b}$ in $\tilde{U}$. The $n+1$ local vector fields $\widetilde{B}_{b}$ and $\widetilde{C}$ form a local field of frames in $\tilde{U}$. At each point of $\tilde{U}$ the coframe $\left\{\zeta^{a}, \tilde{\eta}\right\}$ is dual to the frame $\{\tilde{B} b, \tilde{C}\}$. The equations (2.4) are equivalent to the conditions

$$
\begin{array}{rlrl}
E_{b}^{i} E_{\imath}{ }^{a} & =\delta_{b}^{a}, & E_{b}^{i} E_{\imath}=0, \\
E^{i} E_{\imath}{ }^{a}=0, & E^{i} E_{\imath}=1, & \text { i.e., } \\
\zeta^{a}\left(\tilde{B}_{b}\right) & =\delta_{b}^{a}, & \tilde{\eta}\left(\tilde{B}_{b}\right)=0, \\
\zeta^{a}(\tilde{C})=0, & \tilde{\eta}(\tilde{C})=1
\end{array}
$$

or to the conditions

$$
E_{\imath}{ }^{a} E^{h}{ }_{a}+E_{i} E^{h}=\delta_{i}^{h} .
$$

The first and the second equations of (2.5) or those of (2.5)' show that $n$ local vector fields $\widetilde{B}_{b}$ span the horizontal plane, defined by $\tilde{\eta}=E_{i} d x^{2}=0$, at each point of $\tilde{U}$.

Denoting by $\mathcal{L}$ the Lie derivation with respect to the structure field $\widetilde{C}$, we find $\mathcal{L} E^{h}=0$ and $\mathcal{L} E_{\imath}=0$, which are equivalent to the identity $[\tilde{C}, \tilde{C}]=0$ and the second condition of (1.1) respectively. We also have the relation $\mathcal{L} E_{\imath}{ }^{a}=0$. In fact, as a consequence of (2.2), we find

$$
\partial_{j} E_{\imath}^{a}=\partial_{i} E_{\jmath}{ }^{a}
$$

Thus, taking account of (2.5) and (2.7), we obtain

$$
\begin{aligned}
\mathcal{L} E_{\imath}{ }^{a} & =E^{j} \partial_{j} E_{\imath}{ }^{a}+E_{\jmath}{ }^{a} \partial_{i} E^{\jmath} \\
& =E^{j} \partial_{i} E_{\jmath}{ }^{a}+E_{\jmath}{ }^{a} \partial_{i} E^{\jmath}=\partial_{i}\left(E^{j} E_{\jmath}{ }^{a}\right)=0 .
\end{aligned}
$$

Next, taking Lie derivatives of both sides of the first and the second equations of 
(2. 5), and, also taking account of (2.5) and of the three conditions $\mathcal{L} E^{h}=0, \mathcal{L} E_{\imath}=0$ and $\mathcal{L} E_{\imath}{ }^{a}=0$, we find $\mathcal{L} E^{h}{ }_{b}=0$. Therefore, summing up, we get

$$
\begin{aligned}
\mathcal{L} E^{h}{ }^{h} & =0, & \mathcal{L} E^{h} & =0, \\
\mathcal{L} E_{\imath}{ }^{a} & =0, & \mathcal{L} E_{i} & =0 .
\end{aligned}
$$

Horizontal part. Let there be given a tensor field, say, $\widetilde{T}$ of type $(1,1)$ in the total space $\tilde{M}$. The tensor field $\tilde{T}$ has components of the form

$$
\widetilde{T}_{i}^{h}=T_{b}^{a} E_{i}^{b} E^{h}{ }_{a}+T_{b}{ }^{0} E_{i}^{b} E^{h}+T_{0}^{a} E_{i} E^{h} a+T_{0}{ }^{0} E_{i} E^{h}
$$

with respect to coordinates $\left(x^{h}\right)$ defined in a neighborhood $\tilde{U}$ of $\tilde{M}$, where $T_{b}{ }^{a}, T_{b}{ }^{0}, T_{0}{ }^{a}$ and $T_{0}{ }^{\circ}$ are certain functions in $\tilde{U}$. Thus, taking account of $($ H. 1$) \sim($ H. 4$)$, we easily see that the horizontal part $\widetilde{S}=\tilde{T}^{H}$ of $\tilde{T}$ has components

$$
\tilde{S}_{i}^{h}=T_{b}^{a} E_{i}^{b} E^{h} \text {. }
$$

Invariant function. Let there be given an invariant function $\tilde{f}$ in the total space $\tilde{M}$. We have by definition $\mathcal{L} \tilde{f}=0$, i.e.,

$$
E^{i} \partial_{i} \tilde{f}\left(x^{h}\right)=0
$$

in each neigborhood $\tilde{U}$ in $\tilde{M}$. On the other hand, we see from (2.2) and the second equation of (2.3) that each of the $n$ independent functions $\xi^{a}\left(x^{h}\right)$ appearing in (2.1) is a solution of the partial differential equation

$$
E^{i} \frac{\partial F}{\partial x^{2}}=0
$$

with an unknown function $F$. Therefore, as is well known, the equation (2.9) implies that, for any invariant function $\tilde{f}=\tilde{f}\left(x^{h}\right)$,

$$
\tilde{f}=f\left(\xi^{a}\left(x^{h}\right)\right)
$$

holds in $\tilde{U}$. Taking account of (2.10), we find the formula

$$
\partial_{i} \tilde{f}=E_{\imath}{ }^{a} \partial_{a} f
$$

in $\tilde{U}$ for any invariant function $\tilde{f}$, where the operator $\partial_{a}$ is defined by

$$
\partial_{a}=\frac{\partial}{\partial \xi^{a}}
$$

Let $\tilde{f}$ be an invariant function in $\tilde{M}$ (in $\tilde{U}$ ). Then its projection $f=p \tilde{f}$ is a function in $M$ (in $U=\pi(\tilde{U})$ ) and, conversely, the lift $f^{L}$ of $f$ is equal to $\tilde{f}$. Thus 
in the sequel we shall identify any invariant function $\tilde{f}$ with its projection $f=p \tilde{f}$.

Invariant tensor field, projection and lift. Let there be given an invariant tensor field, say, $\widetilde{T}$ belonging to $\mathcal{G}_{1}^{1}(\tilde{M})$ in the total space $\tilde{M}$. Then, in a neighborhood $\tilde{U}$ of $\tilde{M}, \widetilde{T}$ has components of the form

$$
\widetilde{T}_{i}^{h}=T_{b}^{a} E_{i}^{b} E^{h}{ }_{a}+T_{b}{ }^{0} E_{i}{ }^{b} E^{h}+T_{0}^{a} E_{i} E^{h}{ }_{a}+T_{0}{ }^{0} E_{i} E^{h}
$$

with respect to coordinates $\left(x^{h}\right)$ defined in $\tilde{U}$, where $T_{b}^{a}, T_{b}{ }^{0}, T_{0}{ }^{a}$ and $T_{0}{ }^{0}$ are invariant functions in $\tilde{U}$.

Let there be given an invariant tensor field, say, $\tilde{T}$ belonging to $\mathcal{G}_{1}^{1}(\tilde{M})$. Then $\widetilde{T}$ has components of the form (2.12) with invariant functions $T_{b}^{a}, T_{b}{ }^{0}, T_{0}{ }^{a}$ and $T_{0}{ }^{0}$. Thus, taking account of (P. 1$) \sim(\mathrm{P} .4)$, we can easily see that the projection $T=p \tilde{T}$ has components $T_{b}{ }^{a}$ with respect to coordinates $\left(\xi^{a}\right)$ defined in $\left.U=\pi(\tilde{U}){ }^{5}\right)$

Let there be given a tensor field, say, $T$ of type $(1,1)$ in the base space $M$. Then, taking account of (L. 1$) \sim($ L. 4$)$, we can easily verify that the lift $\widetilde{T}=T^{L}$ of $T$ has components of the form

$$
\widetilde{T}_{i}^{h}=T_{b}^{a} E_{i}^{b} E^{h}{ }_{a}
$$

with respect to coordinates $\left(x^{h}\right)$ defined in neighborhood $\tilde{U}$ of $\tilde{M}$ if the given $T$ has components $T_{b}^{a}=T_{b}{ }^{a}\left(\xi^{c}\right)$ with respect to coordinates $\left(\xi^{a}\right)$ defined in $U=\pi(\tilde{U})$.

Invariant affine connection. Let there be given in the total space $\tilde{M}$ an invariant affine connection $\tilde{\nabla}$ which is torsionless. ${ }^{6)}$ Then, taking a vector field $\tilde{X}$ in $\tilde{M}$, we know by definition that its covariant derivative $\tilde{\nabla} \tilde{X}$ has components of the form

$$
\tilde{\nabla}_{\jmath} \tilde{X}^{h}=\partial_{\jmath} \tilde{X}^{h}+\tilde{\Gamma}_{j}{ }_{i} \tilde{X}^{i}
$$

with respect to coordinates $\left(x^{h}\right)$ defined in each neighborhood $\tilde{U}$ of $\tilde{M}$, where $\tilde{X}^{h}$ and $\tilde{\Gamma}_{j}^{h}$ are respectively components of $\tilde{X}$ and coefficients of $\tilde{\nabla}$ with respect to $\left(x^{h}\right)$ in $\tilde{U}$. Since $\tilde{\nabla}$ is torsionless, we have

$$
\tilde{\Gamma}_{j}{ }_{i}=\tilde{\Gamma}_{i}{ }_{j}{ }_{j}
$$

For each fixed index $a$, the covariant derivative $\tilde{\nabla} \tilde{\zeta}^{a}$ of local covector field $\tilde{\zeta}^{a}$ has components $\tilde{V}_{j} E_{\imath}{ }^{a}$ in a neighborhood $\tilde{U}$ of $\tilde{M}, E_{\imath}{ }^{a}$ being the components of $\tilde{\zeta}^{a}$ in $\tilde{U}$. Because of (2.7) and (2.13), we find

5) The components of a tensor field $T$ in $M$ with respect to coordinates ( $\xi^{a}$ ) defined in $U$, or, the components of $T$ in $U$ mean the components of $T$ with respect to the natural frame $\left\{\partial / \partial \xi^{b}\right\}$ at each point of $U$.

6) Throughout the paper, we shall restrict ourselves to torsionless affine connections in the total space $\tilde{M}$. 


$$
\tilde{\nabla}_{j} E_{\imath}{ }^{a}=\tilde{\nabla}_{i} E_{\jmath}{ }^{a} .
$$

By virtue of (2.4) and $\mathcal{L} E_{\imath}=0$, we can put

$$
\begin{aligned}
\tilde{\nabla}_{j} E_{\imath}{ }^{a} & =-\Gamma_{c}{ }^{a}{ }_{b} E_{\jmath}{ }^{c} E_{i}{ }^{b}+h_{c}{ }^{a} E_{\jmath}{ }^{c} E_{i}+h_{b}{ }^{a} E_{j} E_{i}^{b}+A^{a} E_{j} E_{\imath}, \\
\tilde{\nabla}_{j} E_{\imath} & =-h_{c b} E_{\jmath}{ }^{c} E_{i}{ }^{b}-l_{c} E_{\jmath}{ }^{c} E_{i}-l_{b} E_{j} E_{i}^{b}+D E_{j} E_{\imath}
\end{aligned}
$$

in each neighborhood $\tilde{U}$ of $\tilde{M}$, where

$$
\Gamma_{c}{ }^{a}{ }_{b}=\Gamma_{b}{ }^{a}{ }_{c}
$$

because of (2.14). Taking account of (2. 8), we see that $\Gamma_{c b b}{ }^{a}, h_{c b}, h_{b}{ }^{a}, l_{b}, A^{a}$ and $D$ are all invariant functions in $\tilde{U}$, because the affine connection $\tilde{\nabla}$ is invariant. The first and the second equations of (2.15) are called the co-Gauss equations and the co-Weingarten equations respectively of the given fibred space with an invariant affine connection $\tilde{\nabla}$.

Taking account of (2.5) and (2.15), we find

$$
\begin{gathered}
\tilde{\nabla}_{j} E_{b}^{h}=\Gamma_{c}{ }^{a}{ }_{b} E_{\jmath}{ }^{c} E^{h}{ }_{a}+h_{c b} E_{\jmath}{ }^{c} E^{h}-h_{b}{ }^{a} E_{j} E^{h}{ }_{a}+l_{b} E_{j} E^{h}, \\
\tilde{\nabla}_{j} E^{h}=-h_{c}{ }^{a} E_{\jmath}{ }^{c} E^{h}{ }_{a}+l_{c} E_{\jmath}{ }^{c} E^{h}-A^{a} E_{j} E^{h}{ }_{a}-D E_{j} E^{h} .
\end{gathered}
$$

The invariant functions $\Gamma_{c b}^{a}, h_{c b}, h_{b}^{a}$, etc. are given respectively by the following formulas:

$$
\begin{aligned}
& \Gamma_{c b b}^{a}=-E^{\jmath}{ }_{c} E^{i}{ }_{b} \tilde{\nabla}_{j} E_{\imath}{ }^{a}=E^{\jmath}{ }_{c} E_{\imath}{ }^{a} \tilde{\nabla}_{j} E^{i}{ }_{b}, \\
& h_{c b}=-E^{\jmath}{ }_{c} E^{i}{ }_{b} \tilde{\nabla}_{j} E_{\imath}=-E^{\jmath}{ }_{c} E_{i} \tilde{\nabla}_{j} E^{i}{ }_{b}, \\
& h_{b}{ }^{a}=E^{{ }^{j}}{ }_{b} E^{i} \tilde{\nabla}_{j} E_{\imath}{ }^{a}=E^{j} E^{i}{ }_{b} \tilde{\nabla}_{j} E_{\imath}{ }^{a} \\
& =-E^{j} E_{h}{ }^{a} \tilde{\nabla}_{j} E^{h}{ }_{b}=-E^{\jmath}{ }_{b} E_{h}{ }^{a} \tilde{\nabla}_{j} E^{h},
\end{aligned}
$$

because of (2.15) and (2.17).

Projection of invariant affine connection. Let there be given an invariant affine connection $\tilde{V}$ in a fibred space $\tilde{M}$. Taking an invariant vector field $\tilde{X}$ in $\tilde{M}$, we see that $\tilde{X}$ has components of the form $\tilde{X}^{h}=X^{a} E^{h}{ }_{a}+X^{0} E^{h}$ in each neighborhood $\tilde{U}$ of $\tilde{M}, X^{a}$ and $X^{0}$ being invariant functions in $\tilde{U}$ (cf. (2.12)). Taking account of (2.17), we find that the covariant derivative $\tilde{V} \tilde{X}$ has components of the form

$$
\tilde{\nabla}_{\jmath} \tilde{X}^{h}=\left\{\nabla_{c} X^{a}-X^{0} h_{c}{ }^{a}\right\} E_{\jmath}{ }^{c} E^{h}{ }_{a}+\left\{h_{c a} X^{a}+\left(\partial_{c} X^{0}+l_{c} X^{0}\right)\right\} E_{\jmath}{ }^{c} E^{h}
$$

$$
+\left\{-h_{b}^{a} X^{b}-X^{0} A^{a}\right\} E_{j} E^{h}{ }_{a}+\left\{l_{b} X^{b}-X^{0} D\right\} E_{j} E^{h},
$$


where we have put

$$
\nabla_{c} X^{a}=\partial_{c} X^{a}+\Gamma_{c b}^{a} X^{b} .
$$

When $\tilde{X}$ is invariant and horizontal, the formula (1.29) reduces to the formulas

$$
\tilde{\nabla}_{j} \tilde{X}^{h}=\left(\nabla_{c} X^{a}\right) E_{\jmath}{ }^{c} E^{h}{ }_{a}+\left(h_{c a} X^{a}\right) E_{\jmath}{ }^{c} E^{h}-\left(h_{b}{ }^{a} X^{b}\right) E_{j} E^{h}{ }_{a}+\left(l_{b} X^{b}\right) E_{j} E^{h}
$$

because of $X^{0}=0$. Thus the quantity $h_{c b}$ determines an element $h$ of $\mathscr{I}_{2}^{0}(M)$ and an element $\tilde{h}$ of $\mathcal{G}_{2}{ }_{2}^{\circ}(\tilde{M})$ such that

$$
\begin{aligned}
h(Y, X) & =p\left\{\tilde{\eta}\left(\tilde{\nabla}_{Y L} X^{L}\right)\right\} \quad \text { for } \quad X, Y \in \mathscr{I}_{0}^{1}(M), \\
\tilde{h} & =h^{L} .
\end{aligned}
$$

Similarly, the quantity $h_{b}{ }^{a}$ determines an element $H$ of $\mathscr{I}_{1}^{1}(M)$ and an element $\tilde{H}$ of $\mathcal{g}_{1}^{H_{1}}(\tilde{M})$ such that

$$
\begin{aligned}
H(X) & =-p\left(\tilde{V}_{\widetilde{c}} X^{L}\right) \quad \text { for } \quad X \in \mathscr{I}_{0}^{1}(M), \\
\widetilde{H} & =H^{L} .
\end{aligned}
$$

Taking account of (2.21), we see that the quantity $l_{b}$ determines an element $l$ of $\mathscr{I}_{1}^{0}(M)$ and an element $\tilde{l}$ of $\mathscr{I}_{1}^{H_{1}^{0}}(\tilde{M})$ such that

$$
\begin{aligned}
l(X) & =p\left\{\tilde{\eta}\left(\tilde{\nabla} \tilde{c} X^{L}\right)\right\} \quad \text { for } \quad X \in \mathscr{I}_{1}^{0}(M), \\
\tilde{l} & =l^{L} .
\end{aligned}
$$

By virtue of (2.19), the quantity $A^{a}$ determines an element $A$ of $\mathscr{I}_{0}^{1}(M)$ and an element $\tilde{A}$ of $\mathscr{I}^{H_{0}}(\tilde{M})$ such that

$$
\begin{aligned}
& A=-p\left(\tilde{V}_{\tilde{C}} \tilde{C}\right), \\
& \tilde{A}=A^{L}=-(\tilde{V} \tilde{C} \widetilde{C})^{H}
\end{aligned}
$$

and the invariant function $D$ is expressed by the equation

$$
D=-\tilde{\eta}(\tilde{\nabla} \tilde{C} \tilde{C}) .
$$

The tensor fields $h, H, l, A$ and $D$ defined above have respectively components $h_{c b}, h_{b}{ }^{a}, l_{b}, A^{a}$ and $D$ with respect to coordinates $\left(\xi^{a}\right)$ in $U$. These tensor fields are called the induced tensor fields in $M$.

Let $Y$ and $Z$ be vector fields in the base space $M$. Denote their components in a neighborhood $U=\pi(\tilde{U})$ of $M$ by $Y^{a}$ and $Z^{a}$ respectively, where $\tilde{U}$ is a cylindrical neighborhood of $\tilde{M}$. Then their lifts $Y^{L}$ and $Z^{L}$ have in $\tilde{U}$ components of the form $Y^{h}=Y^{a} E^{h}{ }_{a}$ and $Z^{h}=Z^{a} E^{h}{ }_{a}$ respectively. Therefore, taking account of (2.19), 
we see that the vector field $\widetilde{W}=\tilde{V}_{Y L} Z^{L}$ has in $\tilde{U}$ components of the form

$$
\widetilde{W}^{h}=\left(Y^{c} \nabla_{c} Z^{a}\right) E_{a}^{h}+\left(h_{c b} Y^{c} Z^{b}\right) E^{h},
$$

and hence its projection $W=\nabla_{Y} Z=p\left(\tilde{V}_{Y L} Z^{L}\right)$ has in $U$ components of the form

$$
W^{a}=Y^{c} \nabla_{c} Z^{a}=Y^{c}\left(\partial_{c} Z^{a}+\Gamma_{c}^{a}{ }_{b} Z^{b}\right),
$$

where $\nabla$ is the induced connection in $M$. Consequently, by means of the definition (1.15) of the induced connection, $\nabla_{c} Z^{a}$ appearing in (2.24) are components in $U$ of the covariant derivative $\nabla Z$, where $\nabla$ is the projection of the given invariant affine connection $\tilde{\nabla}$. This fact shows that the induced connection $\nabla$ has coefficients $\Gamma_{c}{ }^{a}$, which appeared in (2.15), with respect to coordinates $\left(\xi^{a}\right)$ in $U$. Thus, taking account of (2.18), we have

Proposition 2.1. Let there be given an invariant and torsionless affine connection $\tilde{\nabla}$ in the total space $\tilde{M}$. Then the connection $\nabla$ induced in the base space $M$ has coefflcients of the form

$$
\begin{aligned}
\Gamma_{c b}{ }_{b} b & =-E^{{ }^{j}}{ }_{c} E^{i_{b}}\left(\partial_{j} E_{\imath}{ }^{a}-\tilde{\Gamma}_{j}{ }^{h}{ }_{i} E_{h}{ }^{a}\right) \\
& =E^{\jmath}{ }_{c} E_{h}{ }^{a}\left(\partial_{j} E^{h}{ }_{b}+\tilde{\Gamma}_{j}{ }^{h}{ }_{i} E^{i}{ }_{b}\right)
\end{aligned}
$$

in each neighborhood $U=\pi(\tilde{U})$, the induced connection $\nabla$ being torsionless, i.e.,

$$
\Gamma_{c b}^{a}=\Gamma_{b}^{a}{ }_{c} .
$$

If we put conventionally in $\tilde{U}$

$$
\stackrel{*}{\nabla}_{j} E_{\imath}{ }^{a}=\tilde{\nabla}_{j} E_{\imath}^{a}+\Gamma_{c}{ }_{b}^{a} E_{\jmath}{ }^{c} E_{i}{ }^{b},
$$

$$
\stackrel{*}{\nabla}_{j} E^{h}{ }_{b}=\tilde{\nabla}_{j} E^{h}{ }_{b}-\Gamma_{c}{ }^{a} E_{j}{ }^{c} E^{h}{ }_{a},
$$

which are the components of $\left(\tilde{\nabla} \tilde{\zeta}^{a}\right)^{V}$ and $\left(\tilde{\nabla} \widetilde{B}_{b}\right)^{V}$ respectively, then the first equations of (2.15) and (2.17) reduce respectively to the equations

$$
\stackrel{*}{\nabla}_{j} E_{\imath}{ }^{a}=h_{c}{ }^{a} E_{j}{ }^{c} E_{i}+h_{b}{ }^{a} E_{j} E_{i}^{b}+A^{a} E_{j} E_{i},
$$

$$
\stackrel{*}{\nabla}_{j} E^{h}{ }_{b}=h_{c b} E_{j}^{c} E^{h}-h_{b}^{a} E_{j} E^{h}+l_{b} E_{j} E^{h} .
$$

Let there be given an element $\tilde{X}$ of $\mathcal{G}_{0}^{H}(\tilde{M})$. Then $\tilde{X}$ has necessarily components $\tilde{X}^{h}=X^{a} E^{h}{ }_{a}$ in each neighborhood $\tilde{U}, X^{a}$ being invariant functions in $\tilde{U}$. Thus, in this case, the formula (2.21) reduces to the formula

$$
\tilde{\nabla}_{\jmath} \tilde{X}^{h}=\left(\nabla_{c} X^{a}\right) E_{\jmath}^{c} E^{h}{ }_{a}+X^{a}{\stackrel{*}{V_{j}}}_{j} E^{h}, \quad \tilde{X}^{h}=X^{a} E^{h}{ }_{a}
$$

because of (2.27). Next, if we suppose that an element of $\mathcal{g}^{H}(\tilde{M})$, say, $\widetilde{T}$ belonging 
to $\mathcal{G}^{H_{1}}(\tilde{M})$ is given in $\tilde{M}$, then $\widetilde{T}$ should have components $\widetilde{T}_{i}{ }^{h}=T_{b}{ }^{a} E_{i}{ }^{b} E^{h}{ }_{a}$ in $\tilde{U}, T_{b}^{a}$ being invariant functions in $\tilde{U}$. We can prove that the covariant derivative $\tilde{\nabla} \tilde{T}$ of $\widetilde{T}$ has componets of the form

$$
\tilde{\nabla}_{\jmath} \widetilde{T}_{i}^{h}=\left(\nabla_{c} T_{b}^{a}\right) E_{\jmath}{ }^{c} E_{i}^{b} E^{h} a+T_{b}^{a}\left(\stackrel{*}{\nabla}_{j} E_{i}^{b}\right) E^{h}{ }_{a}+T_{b}^{a} E_{i}{ }^{b}\left(\stackrel{*}{\nabla}_{j} E^{h}{ }_{a}\right),
$$

where

$$
\nabla_{c} T_{b}^{a}=\partial_{c} T_{b}^{a}+\Gamma_{c}^{a}{ }_{e} T_{b}^{e}-\Gamma_{c}{ }_{b} T_{d}{ }^{a} .
$$

Van der Waerden-Bortolotti covariant derivative. Let there be given an element of the formal tensor product $\mathcal{G}(\tilde{M}) \# \mathcal{G}^{H}(\tilde{M})$, say, $\stackrel{*}{T}$ belonging to $\mathcal{I}_{1}^{1}(\tilde{M}) \# \mathcal{G}_{1}^{H_{1}^{1}}(\tilde{M})$. Then ${ }_{T}^{*}$ is expressed as follows:

$$
\stackrel{*}{T}=T_{j}{ }_{b}{ }^{a} e^{j} \# e_{k} \# \tilde{\zeta}^{b} \# \tilde{B}_{a}
$$

in each cylindrical neighborhood $\tilde{U}$ of $\tilde{M}, T_{j}{ }^{k}{ }^{a}$ being invariant functions in $\tilde{U}$, where $\left\{e_{i}\right\}=\partial / \partial x^{i}$ is the natural frame of coordinates $\left(x^{h}\right),\left\{e^{h}\right\}$ the dual base to $\left\{e_{i}\right\}, \tilde{B}_{a}$ local vector fields having components $E^{h}{ }_{a}$ and $\tilde{\zeta}^{b}$ local covector fields having components $E_{i}{ }^{b}$, all in $\tilde{U}$. We call $T_{j}{ }_{b}{ }^{a}$ the components of $\stackrel{*}{T}$ with respect to coordinates $\left(x^{h}\right)$ and coordinates $\left(\xi^{a}\right)$ defined respectively in $\tilde{U}$ and in $U=\pi(\tilde{U})$, or simply the components of ${ }^{*}$ in $(\tilde{U}, U)$. Let $i: \mathcal{G}(\tilde{M}) \# \mathcal{I}^{H}(\tilde{M}) \rightarrow \mathcal{G}(\tilde{M})$ be the linear homomorphism defined by (I. 1$) \sim($ I. 3$)$ in $\S 1$. Then the image $\tilde{T}=i(\stackrel{*}{T})$ has in $\tilde{U}$ components of the form

$$
\widetilde{T}_{j}{ }_{i}{ }^{h}=T_{j}{ }^{k}{ }^{a} E_{i}^{b} E^{h} a
$$

Conversely, we find

$$
T_{j}{ }^{k}{ }^{a}=\widetilde{T}_{j}{ }^{k}{ }^{h} E^{i}{ }_{b} E_{h}{ }^{a} .
$$

The quantity $E^{h}{ }_{b}$ determines an element $\stackrel{*}{P}$ of $\mathcal{G}_{0}^{1}(\tilde{M}) \# \mathcal{G}_{1}^{H_{1}^{0}}(\tilde{M})$, whose image $\tilde{P}=i(\stackrel{*}{P})$ has components

$$
\begin{aligned}
\tilde{P}_{i}{ }^{h} & =E^{h}{ }_{b} E_{i}{ }^{b} \\
& =\delta_{i}^{h}-E_{i} E^{h}
\end{aligned}
$$

because of (2.6) and (2.30). Therefore $\tilde{P}$ is a projection tensor determining the horizontal plane field. The quantity $E_{\imath}^{a}$ determines an element $\stackrel{*}{Q}$ of $\mathcal{G}_{1}^{0}(\tilde{M}) \# \mathcal{G}^{H}{ }_{0}^{1}(\tilde{M})$ such that $i(\stackrel{*}{Q})=\tilde{P}$.

Let there be given an element of $\mathcal{g}^{H}(\tilde{M})$, say, $\stackrel{*}{S}$ belonging to $\mathcal{G}_{1}^{H_{1}}(\tilde{M})$. Then $\stackrel{*}{S}$ has in $\tilde{U}$ components of the form $\widetilde{S}_{i}{ }^{h}=S_{b}{ }^{a} E_{i}{ }^{b} E^{n} a$ as an element of $\mathcal{G}(\tilde{M})$. Thus, 
taking account of (W. 2), we see that, for any element $\tilde{Y}$ of $\mathcal{G}_{0}^{1}(\tilde{M}), \stackrel{*}{W}=\stackrel{*}{V}_{\tilde{Y}^{H}} \stackrel{*}{S}$ has in $\tilde{U}$ components of the form

$$
\begin{aligned}
{\stackrel{W}{W_{b}}}^{a} & =\left\{\tilde{Y}^{j} \tilde{\nabla}_{j} \tilde{S}_{i}{ }^{h}\right\} E^{i}{ }_{b} E_{h}{ }^{a} \\
& =\left\{\tilde{Y}^{j} \tilde{V}_{j}\left(S_{e}{ }^{d} E_{\imath}{ }^{e} E^{h}{ }_{d}\right)\right\} E^{i}{ }_{b} E_{h}{ }^{a},
\end{aligned}
$$

$\tilde{Y}^{h}=Y^{c} E^{h}{ }_{c}$ being the components of $\tilde{Y}$. This equation reduces to

$$
\stackrel{*}{W}_{b}^{a}=\tilde{Y}^{j} E_{j}{ }^{c} \nabla_{c} S_{b}^{a}=\tilde{Y}^{c} \nabla_{c} S_{b}^{a}
$$

because of (2.27) and (2.29), where

$$
\nabla_{c} S_{b}{ }^{a}=\partial_{c} S_{b}{ }^{a}+\Gamma_{c}{ }^{a}{ }_{e} S_{b}{ }^{e}-\Gamma_{c b}{ }^{e} S_{e}{ }^{a} .
$$

Since the vector field $\tilde{Y}$ in (2.32) is arbitrary, the van der Waerden-Bortolotti covariant derivat!ve $\stackrel{* *}{\nabla S}$ of $\stackrel{*}{S}$ has components of the form

$$
\begin{aligned}
\stackrel{*}{\nabla}_{\jmath} S_{b}{ }^{a} & =E_{\jmath}{ }^{c} \nabla_{c} S_{b}{ }^{a} \\
& =\partial_{\jmath} S_{b}{ }^{a}+E_{\jmath}{ }^{c}\left(\Gamma_{c}{ }_{e}{ }_{e} S_{b}{ }^{e}-\Gamma_{c}{ }^{e}{ }_{b} S_{e}{ }^{a}\right) .
\end{aligned}
$$

Therefore, as a consequence of (W.1) (W. 3), for any element of $\mathcal{G}(\tilde{M}) \# \mathcal{G}^{H}(\tilde{M})$, say, $\stackrel{*}{T}$ belonging to $\mathcal{G}_{1}^{1}(\tilde{M}) \# \mathcal{G}_{1}^{H_{1}}(\tilde{M})$, its van der Waerden-Bortolotti covariant derivative $\stackrel{*}{\nabla} \stackrel{*}{T}$ has components of the form

$$
\begin{aligned}
& { }^{*}{ }_{l} T_{j}{ }_{b}{ }^{a}=\partial_{l} T_{j}{ }_{b}{ }^{a}+\tilde{\Gamma}_{l}{ }_{m}{ }_{m} T_{\jmath}{ }_{b}{ }^{a}-\tilde{\Gamma}_{l}{ }^{m}{ }_{j} T_{m}{ }_{b}{ }^{a} \\
& +E_{l}^{d}\left(\Gamma_{d}^{a}{ }_{e}^{a} T_{\jmath}{ }_{b}^{e}-\Gamma_{d b}^{e} T_{\jmath}{ }^{k}{ }^{a}\right)
\end{aligned}
$$

in $(\tilde{U}, U)$, where $T_{j}{ }^{k}{ }^{a}$ denote the components of $\stackrel{*}{T}$ in $(\tilde{U}, U)$.

The components $\widetilde{T}_{j}{ }_{i}{ }^{h}$ of $\tilde{T}=i(\stackrel{*}{T})$ are given by (2.30) in $\tilde{U}, \stackrel{*}{T}$ being an element of $\mathcal{G}(\tilde{M}) \# \mathcal{G}^{H}(\tilde{M})$ considered above. Taking account of the formula giving $\tilde{\nabla}_{l} \widetilde{T}_{j}{ }^{k}{ }^{h}$, we have the formula

$$
\left.\tilde{\nabla}_{l} \widetilde{T}_{j}{ }_{i}{ }^{h}=\left(\stackrel{*}{\nabla}_{l} T_{j}{ }^{k}{ }_{b}^{a}\right) E_{i}^{b} E^{h}{ }_{a}+T_{j}{ }_{b}{ }^{a}\left(\stackrel{*}{\nabla_{l}} E_{i}^{b}\right) E^{h}{ }_{a}+T_{j}^{k}{ }_{b}^{a} E_{i}^{b} \stackrel{*}{\left(V_{l}\right.} E^{h}{ }_{a}\right)
$$

because of (2.26) and (2.34).

As is well known, we have the Ricci formula

$$
\tilde{V}_{k} \tilde{V}_{j} \tilde{X}^{h}-\tilde{V}_{j} \tilde{V}_{k} \tilde{X}^{h}=\tilde{R}_{k j i}{ }^{h} \tilde{X}^{i}
$$

for any vector field $\tilde{X}$ having components $\tilde{X}^{h}$ in $\tilde{U}$, where $\tilde{R}_{k j i}{ }^{h}$ denote the components of the curvature tensor $\widetilde{R}$ of the given invariant affine connection $\tilde{\nabla}$ and are defined by 


$$
\widetilde{R}_{k j i}{ }^{h}=\partial_{k} \tilde{\Gamma}_{j}{ }_{i}-\partial_{j} \tilde{\Gamma}_{k}{ }_{i}+\tilde{\Gamma}_{k}{ }^{h} \tilde{\Gamma}_{j}{ }_{j}-\tilde{\Gamma}_{j}{ }_{l}{ }_{l} \tilde{\Gamma}_{k}{ }^{l}{ }_{i}
$$

Furthermore, for any element of $\mathcal{G}(\tilde{M}) \# \mathcal{G}^{H}(\tilde{M})$, say, $\stackrel{*}{T}$ belonging to $\mathcal{G}_{0}^{1}(\tilde{M}) \# \mathcal{G}_{1}^{H_{0}^{0}}(\tilde{M})$, which has components $T_{b}^{h}$, we have the formula:

$$
\stackrel{*}{\nabla_{k}} \stackrel{*}{\nabla}_{j} T^{h}{ }_{b}-\stackrel{*}{\nabla} \stackrel{*}{\nabla}_{k} T_{b}^{h}=\widetilde{R}_{k j i}{ }^{h} T_{b}^{i}-E_{k}{ }^{d} E_{j}{ }^{c} R_{d c b}{ }^{a} T^{h}{ }_{a},
$$

where $R_{d c b}{ }^{a}$ denote the components of the curvature tensor $R$ of the induced connection $\nabla$ and are defined by

$$
R_{d c b}^{a}=\partial_{d} \Gamma_{c b}^{a}-\partial_{c} \Gamma_{d b}^{a}+\Gamma_{d}^{a}{ }_{e} \Gamma_{c b}^{e}-\Gamma_{c}^{a}{ }_{e}^{a} \Gamma_{d b}^{e} .
$$

The formula (2.37) is the Ricci formula for van der Waerden-Bortolotti covariant differentiation.

Some formulas. The structure 1 -form $\tilde{\eta}$ has its local expression $\tilde{\eta}=E_{i} d x^{i}$ in each neighborhood $\tilde{U}$ of the total space $\tilde{M}$. Taking account of the second equation of (2.15), we find

$$
d \tilde{\eta}=-\frac{1}{2}\left(h_{c b}-h_{b c}\right) E_{\jmath}^{c} E_{i}^{b} d x^{j} \wedge d x^{i}
$$

Since $d d \tilde{\eta}=0$, we get

$$
\nabla_{d}\left(h_{c b}-h_{b c}\right)+\nabla_{c}\left(h_{b d}-h_{d b}\right)+\nabla_{b}\left(h_{d c}-h_{c d}\right)=0
$$

because of $\stackrel{*}{\nabla}_{j} E_{\imath}^{a}=\stackrel{*}{V}_{i} E_{j}{ }^{a}$. The 2 -form $\Omega$ defined in $M$ by (1.11) is equal to $p(d \tilde{\eta})$. Thus, we have from (2.38)

$$
\Omega=-\frac{1}{2}\left(h_{c b}-h_{b c}\right) d \xi^{c} \wedge d \xi^{b}
$$

The identity (2.39) shows that the 2-form $\Omega$ is closed, where $\Omega$ determines the characteristic class of the given fibred space. (cf. Proposition 1.3).

The horizontal plane field defined by $\tilde{\eta}=0$ is integrable if and only if $d \tilde{\eta} \equiv 0$ $(\bmod \tilde{\eta})$. Thus, taking account of (2.40), we have

Proposition 2.2. The horizontal plane field is integrable if and only if the condition

$$
h_{c b}=h_{b c}
$$

holds.

Let $\widetilde{B}_{b}$ be the local vector fields having components $E^{h_{b}}$ in $\tilde{U}$. Then, taking account of (2.17), we have the formulas: 


$$
\begin{aligned}
{\left[\tilde{B}_{c}, \tilde{B}_{b}\right] } & =\left(h_{c b}-h_{b c}\right) \tilde{C}, \\
{\left[\tilde{C}, \tilde{S}_{b}\right] } & =0 .
\end{aligned}
$$

\section{§ 3. Special cases.}

We discuss, in this section, some of fibred spaces of special type. First, taking account of (2.38), (2.39) and (2.40), we have

Proposition 3.1. When, in an (n+1)-dimensional fibred space $\tilde{M}$, the 2-form $d \tilde{\eta}$ is of the maximum rank $n$, the total space $\tilde{M}$ is necessarily odd-dimensional (i.e., $n+1$ is odd) and the base space $M$ admits a symplectic structure $\Omega=p(d \tilde{\eta})$.

Taking account of (2.17), we have

Proposition 3.2. In a fibred space $\tilde{M}$ with invariant affine connection $\tilde{\nabla}$,

1. $\tilde{\nabla} \tilde{C}=0$, if and only if $h_{b}{ }^{a}, l_{b}, A^{a}$ and $D$ vanish identically.

2. $\tilde{\nabla}_{\tilde{Y}} \tilde{C}=\alpha \tilde{Y}$ for any vector field $\tilde{Y}$ in $\tilde{M}, \alpha$ being an invariant function in $\tilde{M}$, if and only if we have

$$
l_{b}=0, \quad A^{a}=0, \quad h_{b}{ }^{a}=-\alpha \delta_{b}^{a}, \quad D=-\alpha .
$$

3. $\tilde{\nabla}_{\tilde{Y}} \tilde{C}=\alpha \tilde{Y}+\tilde{\omega}(\tilde{Y}) \tilde{C}$ for any vector field $\tilde{Y}$ in $\tilde{M}$, $\alpha$ and $\tilde{\boldsymbol{\omega}}$ being respectively elements of $\mathcal{G}_{0}^{\circ}(\tilde{M})$ and $\mathcal{G}_{1}^{\circ}(\tilde{M})$, if and only if we have

$$
h_{b}{ }^{a}=-\alpha \delta_{b}^{a}, \quad D=-\alpha, \quad \tilde{\omega}_{j}=l_{b} E_{j}^{b}+\alpha E_{\jmath},
$$

where $\tilde{\omega}_{j}$ denote the components of $\tilde{\omega}$.

4. Each fibre is a path if and only if we have $A^{a}=0$.

In a fibred space $\tilde{M}$ with invariant affine connection $\tilde{\nabla}$, let there be given a curve $\tilde{C}$ expressed by equations $x^{h}=x^{h}(t)$ in a neighborhood $\tilde{U}$ of $\tilde{M}, t$ being a parameter. Denoting by $\mathcal{C}$ the image $\pi(\widetilde{C})$ of $\tilde{C}$ by the projection $\pi: \tilde{M} \rightarrow M$, we may assume that $\mathcal{C}$ is expressed by equations $\xi^{a}=\xi^{a}(t)$ in $U=\pi(\tilde{U})$, where the functions $\xi^{a}(t)$ in the right-hand side are defined by $\xi^{a}(t)=\xi^{a}\left(x^{h}(t)\right)$, $\xi^{a}\left(x^{h}\right)$ being the functions appearing in (2.1). In this case, we have

$$
\frac{d \xi^{a}}{d t}=E_{\imath}{ }^{a} \frac{d x^{i}}{d t}
$$

and hence, differentiating covariantly both sides,

$$
\frac{\delta^{2} \xi^{a}}{d t^{2}}=E_{\imath}{ }^{a} \frac{\delta^{2} x^{\imath}}{d t^{2}}+\frac{d \xi^{b}}{d t} E^{\jmath}{ }_{b}\left(\nabla_{j} E_{\imath}{ }^{a}\right) \frac{d x^{\imath}}{d t},
$$

where we have put 


$$
\frac{\delta^{2} x^{h}}{d t^{2}}=\frac{d^{2} x^{h}}{d t^{2}}+\tilde{\Gamma}_{j_{\imath}}{ }_{\imath} \frac{d x^{j}}{d t} \frac{d x^{2}}{d t}, \quad \frac{\delta^{2} \xi^{a}}{d t^{2}}=\frac{d^{2} \xi^{a}}{d t^{2}}+\Gamma_{c}{ }^{a} b \frac{d \xi^{c}}{d t} \frac{d \xi^{b}}{d t}
$$

$\tilde{\Gamma}_{j}{ }^{h}{ }_{\imath}$ and $\Gamma_{c}{ }^{b} a$ being coefficients of the given invariant connection $\tilde{\nabla}$ and its projection $\nabla$ respectively. Substituting $(2.27)$ in (3.1), we have along $\tilde{C}$

$$
\frac{\delta^{2} \xi^{a}}{d t^{2}}=E_{\imath}{ }^{a} \frac{\delta^{2} x^{i}}{d t^{2}}+\left(E_{i} \frac{d x^{2}}{d t}\right) h_{b}{ }^{a} \frac{d \xi^{b}}{d t}
$$

Thus we have

Proposition 3.3. In a fibred space $\tilde{M}$ with invariant affine connection, the projection of any path of $\tilde{M}$ is also a path with respect to the induced connection if and only if

$$
h_{c}^{b}=\alpha \delta_{c}^{b}
$$

$\alpha$ being a certain invariant function in $\tilde{M}$.

A curve $\tilde{C}$ in a fibred space $\tilde{M}$ is said to be horizontal if its tangent vector is horizontal at each point of $\tilde{\mathcal{C}}$. When a curve $\tilde{\mathcal{C}}$ is horizontal in $\tilde{M}$ and a curve $\mathcal{C}$ is the projection $\pi(\tilde{C})$ of $\tilde{C}$ in the base space $M$, the curve $\tilde{C}$ is called a horizontal lift of $C$.

Let $\mathcal{C}$ be a curve in $M$ and be expressed by equations $\xi^{a}=\xi^{a}(t)$ in a neighborhood $U$ of $M$. If a horizontal lift $\tilde{C}$ is expressed by equations $x^{h}=x^{h}(t)$ in a neighborhood $\tilde{U}$ such as $U=\pi(\tilde{U})$, then the functions $x^{h}(t)$ should satisfy the differential equation

$$
\frac{d x^{h}}{d t}=E^{h} \frac{d \xi^{a}}{d t}
$$

Differentiating covariantly both sides, we find along the lift $\tilde{\mathcal{C}}$

$$
\frac{\delta^{2} x^{h}}{d t^{2}}=E^{h} \frac{\delta^{2} \xi^{a}}{d t^{2}}+E^{j}{ }_{b}\left({ }^{*} E_{j}{ }^{h}\right) \frac{d \xi^{b}}{d t} \frac{d \xi^{a}}{d t}
$$

which reduces to

$$
\frac{\delta^{2} x^{h}}{d t^{2}}=E_{a}^{h} \frac{\delta^{2} \xi^{a}}{d t^{2}}+\left(h_{c b} \frac{d \xi^{c}}{d t} \frac{d \xi^{b}}{d t}\right) E^{h}
$$

because of (2.27). Thus we have

Proposition 3.4. In a fibred space $\tilde{M}$ with invariant affine connection, a horizonal curve $\tilde{\mathcal{C}}$ in $\tilde{M}$ is a horizontal path if and only if its projection $\mathcal{C}=\pi(\tilde{\mathcal{C}})$ is a path in the base space $M$ and the condition 


$$
h_{c b} \frac{d \xi^{c}}{d t} \frac{d \xi^{b}}{d t}=0
$$

holds alone $\mathcal{C}$, the equation $\xi^{a}=\xi^{a}(t)$ being the local expression of $\mathcal{C}$. If this is the case, the projection $\pi: \tilde{M} \rightarrow M$ preserves the affine parameters on $\tilde{\mathcal{C}}$ and on $\mathcal{C}$.

Proposition 3.5. In a fibred space $\tilde{M}$ with invariant affine connection, the following two conditions are equivalent to each other:

(a) Any horizontal lift of an arbitrary path given in the base space $M$ is also a path in $\tilde{M}$.

(b)

$$
h_{c b}+h_{b c}=0 \text {. }
$$

In the cases of (a), the projection $\pi: \tilde{M} \rightarrow M$ preserves the affine parameters on the corresponding paths.

Proposition 3.6. Assume that $h_{c b}+h_{b c}=0$ holds in a fibred space $\tilde{M}$ with invariant affine connection. If there are given an arbitrary point $\tilde{\mathrm{P}}$ in $\tilde{M}$ and an arbitrary horizontal vector $\tilde{V}$ at $\tilde{\mathrm{P}}$, then there exists always a unique horizontal path passing through the point $\widetilde{\mathrm{P}}$ and being tangent to the vector $\tilde{V}$ at $\tilde{\mathrm{P}}$.

If this is the case, the projection of any horizontal path is also a path in the base space $M$.

When, in a manifold with an affine connection, any affine parameter of each path takes arbitrary given real value along that path, the manifold is said to be complete (with respect to the affine connection). Thus, taking account of Proposition 3. 6 , we have

Proposition 3.7. Assume that $h_{c b}+h_{b c}=0$ holds in a fibred space $\tilde{M}$ with an invariant affine connection $\tilde{\nabla}$. If the total space $\tilde{M}$ is complete with respect to the affine connection $\tilde{\nabla}$, then the base space $M$ is also complete with respect to the induced affine connection $\nabla$.

\section{$\S 4$. Structure equations.}

In a fibred space $(\tilde{M}, M, \pi ; \tilde{C}, \tilde{\eta})$ with an invariant affine connection $\tilde{\nabla}$, applying the operator $\tilde{V}_{k}$ to both sides of the second equation of (2.26) and taking account of the formulas (2.15), (2.17), (2.27) and (2.34), we have an expression for $\nabla_{k}^{*} \nabla_{j}^{*} E^{h}{ }_{b}$ in terms of $h_{c b}, h_{b}{ }^{a}, l_{b}, A^{a}, D$, their covariant derivatives and $E^{h}, E^{h}, E_{i}, E_{\imath}^{a}$. Thus we have an expression for $\stackrel{*}{V}_{k} \stackrel{*}{V}_{j} E^{h}{ }_{b}-\stackrel{*}{\nabla}_{j}{ }^{*}{ }_{k} E_{b}$ in terms of the quantities mentioned above. If we take account of the Ricci formula (2.37), we find following formulas:

$$
\tilde{R}_{d c b}{ }^{a}=R_{d c b}{ }^{a}-\left(h_{d}{ }^{a} h_{c b}-h_{c}{ }^{a} h_{d b}\right)+\left(h_{d c}-h_{c d}\right) h_{b}{ }^{a},
$$


(4. 1)

$$
\begin{aligned}
& \widetilde{R}_{d o b}{ }^{a}=-\nabla_{d} h_{b}{ }^{a}+l_{d} h_{b}{ }^{a}+h_{d b} A^{a}, \\
& \widetilde{R}_{d c b}{ }^{o}=\left(\nabla_{d} h_{c b}+l_{d} h_{c b}\right)-\left(\nabla_{c} h_{d b}+l_{c} h_{d b}\right)-\left(h_{d c}-h_{c d}\right) l_{b}, \\
& \widetilde{R}_{d o b}{ }^{o}=\left(\nabla_{d} l_{b}+l_{d} l_{b}\right)-h_{d e} h_{b}{ }^{e}+D h_{d b},
\end{aligned}
$$

where we have put

$$
\begin{array}{ll}
\widetilde{R}_{d c b}{ }^{a}=E_{d}^{k} E^{\jmath}{ }_{c} E_{b}^{i} E_{h}{ }^{a} \widetilde{R}_{k j i}{ }^{h}, & \widetilde{R}_{d o b}{ }^{a}=E_{d}^{k} E^{j} E_{b}{ }_{b} E_{h}{ }^{a} \widetilde{R}_{k j i}{ }^{h}, \\
\widetilde{R}_{d c b}{ }^{o}=E^{k}{ }_{d} E^{\jmath} E^{i} E_{b} E_{h} \widetilde{R}_{k j i}{ }^{h}, & \widetilde{R}_{d o b}{ }^{o}=E^{k}{ }_{d} E^{j} E^{i} E_{h} \tilde{R}_{k j i}{ }^{h} .
\end{array}
$$

Similarly, applying the operator $\tilde{\nabla}_{k}$ to both sides of the second equation of (2.17), we have an expression for $\tilde{\nabla}_{k} \tilde{\nabla}_{j} E^{h}$ in terms of $h_{c b}, h_{b}{ }^{a}, \cdots$ and $E^{h}, E^{h}, \cdots$. Thus we have an expression for $\tilde{\nabla}_{k} \tilde{V}_{j} E^{h}-\tilde{\nabla}_{j} \tilde{\nabla}_{k} E^{h}$ in terms of the quantities mentioned above. If we take account of the Ricci formula (2.36), we find following formulas:

$$
\begin{aligned}
& \tilde{R}_{d c o}{ }^{a}=-\left(\nabla_{d} h_{c}{ }^{a}-l_{d} h_{c}{ }^{a}\right)+\left(\nabla_{c} h_{d}{ }^{a}-l_{c} h_{d}{ }^{a}\right)+\left(h_{d c}-h_{c d}\right) A^{a}, \\
& \tilde{R}_{d o o}{ }^{a}=-\nabla_{d} A^{a}-h_{d}{ }^{e} h_{e}{ }^{a}+l_{d} A^{a}+D h_{d}{ }^{a}, \\
& \tilde{R}_{d c o}{ }^{o}=\left(\nabla_{d} l_{c}-\nabla_{c} l_{d}\right)-\left(h_{d e} h_{c}^{e}-h_{c e} h_{d}^{e}\right)+D\left(h_{d c}-h_{c d}\right), \\
& \tilde{R}_{d o o}^{o}=-\partial_{d} D-h_{d e} A^{e}+h_{d}^{e} l_{e},
\end{aligned}
$$

where we have put

$$
\begin{array}{ll}
\widetilde{R}_{d c o}{ }^{a}=E^{k}{ }_{d} E_{c}^{j} E^{i} E_{h}{ }^{a} \widetilde{R}_{k j i}{ }^{h}, & \widetilde{R}_{d o o}{ }^{a}=E^{k}{ }_{d} E^{j} E^{i} E_{h}{ }^{a} \widetilde{R}_{k j i}{ }^{h}, \\
\widetilde{R}_{d c o}{ }^{o}=E^{k}{ }_{d} E_{c}^{j} E^{i} E_{h} \widetilde{R}_{k j i}{ }^{h}, & \widetilde{R}_{d o o}{ }^{o}=E^{k}{ }_{d} E^{j} E^{i} E_{h} \widetilde{R}_{k j i}{ }^{h} .
\end{array}
$$

The formulas (4.1) and (4.2) form the so-called structure equations of the given fibred space with invariant affine connection. They correspond to those of Gauss, Codazzi and Ricci in the theory of subspaces.

When the invariant affine connection $\tilde{V}$ is of zero curvature in a fibred space $\tilde{M}$, we have eight equations by putting $\widetilde{R}_{k j i}{ }^{h}=0$ in (4.1) and (4.2). However, among these eight equations, only the following six equations are independent. Therefore, the structure equations:

$$
\begin{aligned}
& R_{d c b}{ }^{a}=\left(h_{d}{ }^{a} h_{c b}-h_{c}{ }^{a} h_{d}^{b}\right)-\left(h_{d c}-h_{c d}\right) h_{b}{ }^{a}, \\
& \nabla_{d} h_{b}{ }^{a}-l_{d} h_{b}{ }^{a}-h_{d b} A^{a}=0, \\
& \nabla_{d} A^{a}+h_{d}{ }^{e} h_{e}{ }^{a}-l_{d} A^{a}-D h_{d}{ }^{a}=0, \\
& \left(\nabla_{d} h_{c b}+l_{d} h_{c b}\right)-\left(\nabla_{c} h_{d b}+l_{c} h_{d b}\right)-\left(h_{d c}-h_{c d}\right) l_{b}=0,
\end{aligned}
$$




$$
\begin{aligned}
& \nabla_{d} l_{b}+l_{d} l_{b}-h_{d e} h_{b}{ }^{e}+D h_{d b}=0, \\
& \partial_{d} D+h_{d e} A^{e}-h_{d}^{e} l_{e}=0
\end{aligned}
$$

hold in the base space $M$ with respect to the induced connection $\nabla$, if the invarnant affine connection $\tilde{\nabla}$ is of zero curvature in the total space $\tilde{M}$

\section{$\S 5$. The fibering in flat spaces.}

Let $M$ be an $n$-dimensional differentiable manifold with a torsionless affine connection $\nabla$. Assume that there exist in $M$ five tensor fields $h_{c b}, h_{b}{ }^{a}, l_{b}, A^{a}$ and $D$ of type $(0,2),(1,1),(0,1),(1,0)$ and $(0,0)$, respectively. Denote by $R^{n+1}$ the affine space of $n+1$ dimensions with cartesian coordinates $\left(x^{h}\right)=\left(x^{1}, x^{2}, \cdots, x^{n+1}\right)$. The natural affine connection $\tilde{\nabla}$ of $R^{n+1}$ is torsionless and of zero curvature. We suppose that there exists in $R^{n+1}$ an open set $\tilde{U}$, a differentiable mapping $\pi: \tilde{U} \rightarrow M$ of the maximum $\operatorname{rank} n$, a vector field $\tilde{C}$ in $\tilde{U}$ and a 1 -form $\tilde{\eta}$ in $\tilde{U}$ such that the set $(\tilde{U}, M, \pi ; \tilde{C}, \tilde{\eta})$ defines a fibred space with $\tilde{V}$ as its invariant affine connection, and, such that the affine connection $\nabla$ and the five tensor fields $h_{c b}, h_{b}{ }^{a}, l_{b}, A^{a}$ and $D$ given in $M$ are all induced from the structure of the fibred space $(\tilde{U}, M, \pi ; \widetilde{C}, \tilde{\eta})$. If this is the case, we say that there exists in $\tilde{U}$ a fibering which is compatible with the structure $\left(\nabla, h_{c b}, h_{b}{ }^{a}, l_{b}, A^{a}, D\right)$ over $M$.

We shall show in this section that, for a differentiable manifold $M$ with a structure $\left(\nabla, h_{c b}, h_{b}{ }^{a}, l_{b}, A^{a}, D\right)$, there exists in a certain open set $\tilde{U}$ of $R^{n+1}$ a fibering over $M$, which is compatible with the structure given in $M$, if the structure given in $M$ satisfies the conditions (4.3) and $M$ is a sufficiently small $n$-cube.

Let $M$ be an $n$-cube with coordinates $\left(\xi^{a}\right)=\left(\xi^{1}, \xi^{2}, \cdots, \xi^{n}\right)\left(\left|\xi^{a}\right|<\delta^{a}, \delta^{a}>0, a=1,2, \cdots, n\right)$ and $\nabla$ a torsionless affine connection whose coefficients are denoted by $\Gamma_{c b}{ }_{b}$ with respect to coordinates $\left(\xi^{a}\right)$, where $\Gamma_{c}{ }^{a}{ }_{b}=\Gamma_{b}{ }^{a}{ }_{c}$. Assume moreover that there are given in $M$ five tensor fields $h_{c b}, h_{b}^{c}, l_{b}, A^{a}$ and $D$ of the corresponding type, where these symbols denote respectively the components of the corresponding tensor fields with respect to coordinates $\left(\xi^{a}\right)$, and these five tensor fields satisfy the conditions (4.3). We consider now the following partial differential equations:

$$
\begin{aligned}
\frac{\partial E_{\imath}{ }^{a}}{\partial x^{\jmath}} & =-\Gamma_{c b}^{a} E_{\jmath}{ }^{c} E_{i}^{b}+h_{c}{ }^{a} E_{\jmath}{ }^{c} E_{i}+h_{b}{ }^{a} E_{j} E_{i}{ }^{b}+A^{a} E_{j} E_{\imath}, \\
\frac{\partial E_{i}}{\partial x^{\jmath}} & =-h_{c b} E_{\jmath}{ }^{c} E_{i}{ }^{b}-l_{c} E_{\jmath}{ }^{c} E_{i}-l_{b} E_{j} E_{i}^{b}+D E_{j} E_{\imath}, \\
\frac{\partial \xi^{a}}{\partial x^{\jmath}} & =E_{\jmath}{ }^{a}
\end{aligned}
$$

with unknown functions $\xi^{a}\left(x^{h}\right), E_{\imath}{ }^{a}\left(x^{h}\right)$ and $E_{i}\left(x^{h}\right),\left(x^{h}\right)$ being the independent variables, where $\Gamma_{c}{ }^{a} b, h_{c b}, h_{b}{ }^{a}, l_{b}, A^{a}$ and $D$ appearing in (5.1) are given functions depending on the unknown variables $\left(\xi^{a}\right)$. It is easily verified that the integrability 
condition of the partial differential equations (5.1) is the condition (4. 3). Thus the partial differential equations (5.1) are completely integrable, because the condition (4. 3) is assumed to be satisfied by the given $\Gamma_{c}^{b}{ }_{a} a, h_{c b}, h_{b}{ }^{a}, l_{b}, A^{a}$ and $D$.

Let a solution $\left(\xi^{a}, E_{\imath}{ }^{a}, E_{i}\right)$ of $(5.1)$ be given. Denote by $\Delta$ the determinant

$$
\Delta=\left|E_{i}^{a}, E_{i}\right| \text {. }
$$

If we take account of (5.1), we easily verify that the determinant $\Delta$ satisfies the differential equations

$$
\frac{\partial \Delta}{\partial x^{j}}=\left[-E_{j}^{c}\left(\Gamma_{c a}^{a}+l_{c}\right)+E_{j}\left(h_{a}^{a}+D\right)\right] 4
$$

Take an arbitrary point $\widetilde{\mathrm{P}}_{0}$ with coordinates $\left(\dot{x}^{h}\right)$ in $R^{n+1}$ and $(n+1)^{2}$ values

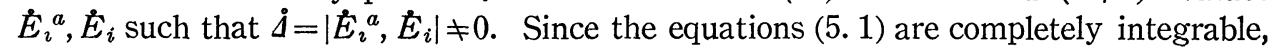
there exists one and only one set of solutions $\left(\xi^{a}\left(x^{h}\right), E_{\imath}{ }^{a}\left(x^{h}\right), E_{i}\left(x^{h}\right)\right)$ of $(5.1)$, which satisfies the initial conditions

$$
\xi^{a}\left(\dot{x}^{h}\right)=0, \quad E_{\imath}^{a}\left(\dot{x}^{h}\right)=\dot{E}_{\imath}{ }^{a}, \quad E_{i}\left(\dot{x}_{\imath}^{h}\right)=\dot{E}_{\imath},
$$

where the solution $\left(\xi^{a}\left(x^{h}\right), E_{\imath}^{a}\left(x^{h}\right), E_{i}\left(x^{h}\right)\right)$ is defined in a certain cubic neighborhood $\tilde{U}$ containing the point $\widetilde{\mathrm{P}}_{0}$. The function $\Delta\left(x^{h}\right)$ defined by $(5.2)$ is a solution of a homogeneous differential equation (5.3). Thus $\Delta\left(x^{h}\right)$ does not vanish anywhere in $\tilde{U}$, because $A\left(\dot{x}^{h}\right)=\left|\dot{E}_{i}{ }^{h}, E_{i}\right| \neq 0$ holds by virtue of the assumption. We denote by $\tilde{\zeta}^{a}$ a covector field having components $E_{\imath}{ }^{a}\left(x^{h}\right)$ for each fixed index $a$ and by $\tilde{\eta}$ a covector field having components $E_{i},\left(x^{h}\right)$ all in $\tilde{U}$. Then the $n+1$ covector fields $\tilde{\zeta}^{a}$ and $\tilde{\eta}$ thus introduced are linearly independent at each point of $\tilde{U}$, because $\Delta=\left|E_{i}^{a}, E_{i}\right| \neq 0$ as is mentioned above. Taking account of (5.1), we find $\partial \xi^{a} / \partial x^{\imath}=E_{\imath}{ }^{a}$ for the solution $\left(\xi^{a}\left(x^{h}\right), E_{\imath}^{a}\left(x^{h}\right), E_{i}\left(x^{h}\right)\right)$. Since the matrix $\left(E_{\imath}^{a}\right)=\left(\partial \xi^{a} / \partial x^{i}\right)$ is of the maximum rank $n$, the correspondence

$$
\pi:\left(x^{h}\right) \rightarrow\left(\xi^{a}\left(x^{h}\right)\right)
$$

defines a differentiable mapping $\pi$ of $\tilde{U}$ into $M$ and $\pi$ is of the maxiuum rank, the open set $\tilde{U}$ being taken, if necessary, sufficiently small. The image $\pi(\tilde{U})$ is an open set in $M$. For the sake of simplicity, we denote $\pi(\tilde{U})$ simply by $M$.

Summing up, we have proved the fact that, given in $R^{n+1}$ an arbitrary point $\widetilde{\mathrm{P}}_{0}$ and $n+1$ linearly independent covectors $\mathscr{E}_{i}{ }^{a}, \mathscr{E}_{i}$ at that point $\widetilde{\mathrm{P}}_{0}$, then there exist in $R^{n+1}$ a cubic open set $\tilde{U}$ containing $\widetilde{\mathrm{P}}_{0}$, an onto differentiable mapping $\pi$ : $\tilde{U} \rightarrow M$ and $n+1$ linearly independent covector fields $E_{\imath}{ }^{a}, E_{i}$ defined in $\tilde{U}$ such that $E_{i}{ }^{h}=E_{i}{ }^{h}$, $E_{2}=\dot{E}_{i}$ at $\widetilde{\mathrm{P}}_{0}$ and $\pi\left(\widetilde{\mathrm{P}}_{0}\right)=0$, where $\mathrm{O}$ is the point with coordinates $(0,0, \cdots, 0)$ in $M$.

We now construct in $\tilde{U} n+1$ vector fields $\widetilde{B}_{b}$ and $\tilde{C}$ having respectively components $E^{h}{ }_{b}$ and $E^{h}$ such that 


$$
\left(\begin{array}{c}
E^{h} b \\
E^{h}
\end{array}\right)=\left(E_{\imath}^{a}, E_{i}\right)^{-1} \text {. }
$$

Then we get (2.5) (or $(2.5)^{\prime}$ ) and (2.6). Moreover, the vector field $\widetilde{C}$ thus defined is tangent to the fibre at each point of $\tilde{U}$, where a fibre is by definition the inverse image $\pi^{-1}(\mathrm{P})$ of $\mathrm{P}$ in $\tilde{U}, \mathrm{P}$ being an arbitrary point of $M$. In the next step, denoting by $\mathcal{L}$ the Lie derivation with respect to $\widetilde{C}$, we easily find $\mathcal{L} E^{h}=0$ and $\mathcal{L} E_{\imath}{ }^{a}=0$. On the other hand, taking account of (2.5) and (5.1), we obtain the equations

$$
\begin{aligned}
& \frac{\partial E^{h}{ }_{b}}{\partial x^{\jmath}}=\Gamma_{c}{ }_{b}{ }_{b} E_{\jmath}{ }^{c} E^{h}{ }_{a}+h_{c b} E_{\jmath}{ }^{c} E^{h}-h_{b}{ }^{a} E_{j} E^{h}{ }_{a}+l_{b} E_{j} E^{h}, \\
& \frac{\partial E^{h}}{\partial x^{\jmath}}=-h_{c}{ }^{a} E_{\jmath}{ }^{c} E^{h}{ }_{a}+l_{c} E_{\jmath}{ }^{c} E^{h}-A^{a} E_{j} E^{h}{ }_{a}-D E_{j} E^{h} .
\end{aligned}
$$

Thus, by means of (2.5), (5.1) and the second equation of (5.6), we find

$$
\mathcal{L} E_{\imath}=E^{j} \partial_{j} E_{i}+E_{j} \partial_{i} E^{\jmath}=0 .
$$

Therefore, taking account of (2.5), we get $\mathcal{L} E^{h}{ }_{b}=0$ because of $\mathcal{L} E_{\imath}{ }^{a}=0$ and $\mathcal{L} E_{\imath}=0$. Consequently, we have $\mathcal{L} E_{\imath}{ }^{a}=0, \mathcal{L} E_{\imath}=0, \mathcal{L} E^{h}{ }_{b}=0$ and $\mathcal{L} E^{h}=0$. Thus, summing up, we see that the set $(\tilde{U}, M, \pi ; \tilde{C}, \tilde{\eta})$ is a fibred space.

We shall now show that the natural affine connection $\tilde{\nabla}$ of $R^{n+1}$ is invariant under the infinitesimal transformation determined by $\tilde{C}$. If we denote in $\widetilde{U}$ the images by $*_{\pi}$ of the functions $\Gamma_{\mathrm{c}}{ }^{a} \mathrm{~b}, h_{\mathrm{c} b}, h_{b}{ }^{a}, l_{b}, A^{a}$ and $D$ by the same corresponding symbols, we find that all of their Lie derivatives $\mathcal{L} \Gamma_{c}{ }^{a}, \mathcal{L} h_{c b}, \cdots, \mathcal{L} D$ vanish identically in $\tilde{U}$. Therefore, applying the operator $\mathcal{L}$ to both sides of the two equations of (5.6), we have

$$
\mathcal{L}\left(\frac{\partial E^{h} b}{\partial x^{j}}\right)=0, \quad \mathcal{L}\left(\frac{\partial E^{h}}{\partial x^{j}}\right)=0
$$

because of $\mathcal{L} E_{\imath}{ }^{a}=0, \mathcal{L} E_{\imath}=0, \mathcal{L} E^{h}{ }_{b}=0$ and $\mathcal{L} E^{h}=0$. These facts show that the vector field $\widetilde{C}$ leaves in $R^{n+1}$ the natural affine connection $\tilde{V}$ invariant. Consequently, the fibred space $(\tilde{U}, M, \pi ; \tilde{C}, \tilde{\eta})$ has the natural affine connection $\tilde{V}$ as its invariant affine connection. Thus we have

Proposition 5.1. Let $M$ be a differentiable manifold, which is a sufficiently small $n$-cube, and there be given a torsionless affine connection $\nabla$ and five tensor fields $h_{c b}, h_{b}{ }^{a}, l_{b}, A^{a}, D$ of the type $(0,2),(1,1),(1,0),(0,1),(0,0)$, respectively in $M$. Assume that $\nabla, h_{c b}, h_{b}{ }^{a}, l_{b}, A^{a}$ and $D$ satisfy the condition (4. 3). Given a point $\mathrm{O}$ in $M$ and a point $\widetilde{\mathrm{P}}_{0}$ in $R^{n+1}$, then there exists in $R^{n+1}$ an open set $\widetilde{U}$ containing $\widetilde{\mathrm{P}}_{0}$, an onto differentiable mapping $\pi: \tilde{U} \rightarrow M$ of the maximum rank $n$, a vector field $\tilde{C}$ in $\tilde{U}$ and a 1-form $\tilde{\eta}$ in $\tilde{U}$ such that the set $(\tilde{U}, M, \pi ; \tilde{C}, \tilde{\eta})$ is a fibred space having the natural connection of $R^{n+1}$ as its invariant affine connection and the fibering 
determined in $\tilde{U}$ by the fibred space $(\tilde{U}, M, \pi ; \tilde{C}, \tilde{\eta})$ is compatible with the given structure $\left(\nabla, h_{c b}, h_{b}{ }^{a}, l_{b}, A^{a}, D\right)$ of $M$. Such a fibering is determined by giving $n+1$ linearly independent covector $\dot{E}_{\imath}{ }^{a}, \dot{E}_{\imath}$ at the point $\widetilde{\mathrm{P}}_{0}$.

Let there be given an affine transformation $\mathcal{A}$ of $R^{n+1}$, which is represented by equations

$$
' x^{h}=\sum_{k=1}^{n+1} a_{k}^{h} x^{k}+a^{h}, \quad\left|a_{k}^{h}\right| \neq 0,
$$

$a_{k}^{h}$ and $a^{h}$ being constant, where $\left(x^{h}\right)$ and $\left({ }^{\prime} x^{h}\right)$ are respectively coordinates of a point $\widetilde{\mathrm{P}}$ and of its image $\mathcal{A}(\widetilde{\mathrm{P}})$ in $R^{n+1}$. We now put

$$
'^{\prime}{ }^{a}=\sum_{n=1}^{n+1} b_{i}^{k} \dot{E}_{k}{ }^{a}, \quad ' \dot{E}_{\imath}=\sum_{n=1}^{n+1} b_{i}^{k} \dot{E}_{k}, \quad ' \dot{x}^{n}=\sum_{k=1}^{n+1} a_{k}^{h} \dot{x}^{k}+a^{h},
$$

the matrix $\left(b_{i}^{h}\right)$ being the inverse of the matrix $\left(a_{i}^{h}\right)$. If we denote by $\left({ }^{\prime} \xi^{a}\left(x^{h}\right)\right.$, $\left.{ }^{\prime} E_{\imath}^{a}\left(x^{h}\right), E_{i}\left(x^{h}\right)\right)$ the solution of the differential equation (5.1) which satisfies the initial conditions

$$
{ }^{\prime} \xi^{a}\left({ }^{\prime} \dot{x}^{h}\right)=0, \quad ' E_{\imath}{ }^{a}\left(\dot{x}^{h}\right)=\prime^{\prime}{ }_{\imath}{ }^{a}, \quad ' E_{i}\left({ }^{\prime} \dot{x}^{h}\right)={ }^{\prime} E_{\imath},
$$

then we have

$$
{ }^{\prime} E_{\imath}{ }^{a}=\sum_{k=1}^{n} b_{i}^{k} E_{k}^{a}, \quad ' E_{\imath}=\sum_{k=1}^{n} b_{i}^{k} E_{k}, \quad ' \xi^{a}\left(x^{h}\right)=\xi^{a}\left(\sum_{k=1}^{n} a_{k}^{h} x^{k}+a^{h}\right) .
$$

Moreover there exists a fibred space $\left({ }^{\prime} \tilde{U}, M{ }^{\prime} \pi ;^{\prime} \tilde{C}, ' \tilde{\eta}\right)$ with the natural affine connection $\tilde{V}$ of $R^{n+1}$ as its invariant affine connection and the fibering determined by the new fibred space is compatible with the structure $\left(\nabla, h_{c b}, h_{b}{ }^{a}, l_{b}, A^{a}, D\right)$ given in $M$, where $' \tilde{U}=\mathcal{A}(\tilde{U}),{ }^{\prime} \pi=\pi \circ \mathcal{A}^{-1}, ' \widetilde{C}=\mathcal{A}(\tilde{C})$ and ' $\tilde{\eta}=\mathcal{A}(\tilde{\eta})$. The new fibering is called the fibering transformed by the affine transformation $\mathcal{A}$ of the old fibering determined by the fibred space $(\tilde{U}, M, \pi ; \tilde{C}, \tilde{\eta})$. Thus we have

Proposition 5.2. The fibering, whose existence is proved in Proposition 5.1, is determined up to affine transformations in the affine space $R^{n+1}$.

\section{§6. Manifolds with projective connection.}

Let $(\tilde{M}, M, \pi ; \tilde{C}, \tilde{\eta})$ be a fibred space with an invariant affine connection $\tilde{\nabla}$, which is torsionless. Assume that the structure field $\widetilde{C}$ satisfies the condition

$$
\tilde{\nabla}_{\tilde{Y}} \tilde{C}=\alpha \tilde{Y} \quad \text { for } \quad \tilde{Y} \in \mathcal{I}_{0}^{1}(\tilde{M}),
$$

$\alpha$ being a non-zero constant. Then, by virtue of Proposition 3.2, we find

$$
h_{b}{ }^{a}=-\alpha \delta_{b}^{a}, \quad l_{c}=0, \quad A^{a}=0, \quad D=-\alpha
$$


in each cylindrical coordinate $\tilde{U}$ of the total space $\tilde{M}$. Therefore, the equations (2.15) and (2.17) reduce to

$$
\begin{aligned}
\tilde{\nabla}_{j} E_{\imath}{ }^{a} & =-\Gamma_{c}{ }^{a}{ }_{b} E_{\jmath}{ }^{c} E_{i}{ }^{b}-\alpha E_{\jmath}{ }^{a} E_{i}-\alpha E_{j} E_{\imath}{ }^{a}, \\
\tilde{\nabla}_{j} E_{\imath} & =-h_{c b} E_{\jmath}{ }^{c} E_{i}{ }^{b}-\alpha E_{j} E_{\imath}, \\
\tilde{\nabla}_{j} E^{h}{ }_{b} & =\Gamma_{c}{ }_{c}{ }_{b} E_{\jmath}{ }^{c} E^{h}{ }_{a}+h_{c b} E_{\jmath}{ }^{c} E^{h}+\alpha E_{j} E^{h}{ }_{b}, \\
\tilde{\nabla}_{j} E^{h} & =\alpha \delta_{\jmath}^{h} .
\end{aligned}
$$

Change of horizontal planes. Let there be given a 1 -form $\tilde{\phi}$ in the total space $\tilde{M}$ such that

$$
\mathcal{L} \tilde{\phi}=0, \quad \tilde{\phi}(\tilde{C})=0
$$

If we put

$$
{ }^{\prime} \tilde{\eta}=\tilde{\eta}-\tilde{\phi},
$$

then the set $\left(\tilde{M}, M, \pi ; \tilde{C},{ }^{\prime} \tilde{\eta}\right)$ defines a new fibred space, which is said to be obtained by change (6.4) of horizontal planes from the original fibred space $(\tilde{M}, M, \pi ; \widetilde{C}, \tilde{\eta})$. If we put in each cylindrical neighborhood $\tilde{U}$ of $\tilde{M}$

$$
\begin{array}{ll}
\bar{E}_{\imath}^{a}=E_{\imath}{ }^{a}, & \bar{E}_{\imath}=E_{i}-\phi_{i}, \\
\bar{E}^{h}{ }_{b}=E^{h}{ }_{b}+\phi_{b} E^{h}, & \bar{E}^{h}=E^{h},
\end{array}
$$

invariant functions $\phi_{b}$ being defined by $\phi_{b}=E^{i}{ }^{i} \phi_{i}$ and $\phi_{i}$ being components of the vector field $\tilde{\phi}$ in $\tilde{U}$, then we find

$$
\left(\begin{array}{c}
\bar{E}^{h} \\
\bar{E}^{h}
\end{array}\right)=\left(\bar{E}_{i}^{b}, \bar{E}_{\imath}\right)^{-1}
$$

and

$$
\begin{aligned}
\mathcal{L} \bar{E}^{h} b & =0, \\
\mathcal{L} \bar{E}_{\imath}^{a}=0, & \mathcal{L} \bar{E}^{h}=0, \\
\mathcal{L} \bar{E}_{i} & =0,
\end{aligned}
$$

because of (6.3) and (6.4), where $\bar{E}^{h}\left(=E^{h}\right)$ and $\bar{E}_{\imath}$ are components of $\tilde{C}$ and ' $\tilde{\eta}$ in $\tilde{U}$ respectively. If we differentiate covariantly both sides of the first two equations of (6.6) and take account of (6.2), we obtain

$$
\begin{aligned}
\tilde{\nabla}_{j} \bar{E}_{\imath}^{a} & =-\left(\Gamma_{c b}^{a}+\alpha \phi_{c} \delta_{b}^{a}+\alpha \phi_{b} \delta_{c}^{a}\right) \bar{E}_{\jmath}^{c} \bar{E}_{i}^{b}-\alpha \bar{E}_{\jmath}^{a} \bar{E}_{i}-\alpha \bar{E}_{j} \bar{E}_{\imath}^{a}, \\
\tilde{\nabla}_{j} \bar{E}_{\imath} & =-\left(h_{c b}+\nabla_{c} \phi_{b}-\alpha \phi_{c} \phi_{b}\right) \bar{E}_{\jmath}^{c} \bar{E}_{i}^{b}-\alpha \bar{E}_{j} \bar{E}_{\imath}
\end{aligned}
$$

because of (6.6). 
Denote by $\left(\nabla, h_{c b}\right)$ and $\left({ }^{\prime} \nabla,{ }^{\prime} h_{c b}\right)$ repsectively the structure induced in the base space $M$ from the fibred spaces $(\tilde{M}, M, \pi ; \tilde{C}, \tilde{\eta})$ and $\left(\tilde{M}, M, \pi ; \tilde{C},{ }^{\prime} \tilde{\eta}\right)$. Then we have

$$
{ }^{\prime} \Gamma_{\mathrm{c} b}^{a}=\Gamma_{\mathrm{c} b}^{a}+\alpha \phi_{\mathrm{c}} \delta_{b}^{a}+\alpha \phi_{b} \delta_{\mathrm{c}}^{a},
$$

$$
h_{c b}=h_{c b}+\nabla_{c} \phi_{b}-\alpha \phi_{c} \phi_{b}
$$

as a consequence of (6.7), $\phi_{b}\left(=E_{b}^{i} \phi_{i}\right)$ being components of the projection $p \tilde{\phi}$ in $U=\pi(\tilde{U})$ with respect to old fibred space $(\tilde{M}, M, \pi ; \widetilde{C}, \tilde{\eta})$, where $\Gamma_{c}{ }^{a} b$ and ' $\Gamma_{c}{ }^{a} b$ denote

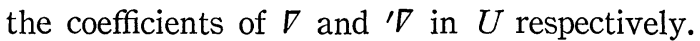

Thus, taking account of the first equation of (6.8), we have

Proposition 6.1. If there is given an invariant affine connection $\tilde{\nabla}$ satisfying the condition (6.1) in a fibred space without horizontal planes, then any two connections $\nabla$ and ' $\nabla$, which are induced in the base space $M$ by giving structure 1-forms $\tilde{\eta}$ and ' $\tilde{\eta}$ in $\tilde{M}$ respectively, are projectively related to each other. That is to say, if there is given an invariant affine connection $\tilde{\nabla}$ in a fibred space $\tilde{M}$ without horizontal planes, a projective structure is induced in the base space $M$, the induced projective structure being determined by an affine connection $\nabla$ induced in $M$ by giving a structure 1-form $\tilde{\eta}$ in $\tilde{M}$.

Coming back to a fibred space $(\tilde{M}, M, \pi ; \tilde{C}, \tilde{\eta})$ with invariant affine connection $\tilde{\nabla}$ satisfying the condition (6.1), we find from the structure equations (4.1) and (4.2)

$$
\widetilde{R}_{d c b}{ }^{a}=R_{d c b}{ }^{a}+\alpha\left[\left(\delta_{d}^{a} h_{c b}-\delta_{c}^{a} h_{d b}\right)-\left(h_{d c}-h_{c d}\right) \delta_{b}^{a}\right]
$$

$$
\widetilde{R}_{d c b}{ }^{o}=\nabla_{a} h_{c b}-\nabla_{c} h_{d b}
$$

and

$$
\begin{array}{lll}
\widetilde{R}_{d o b}{ }^{a}=0, & \widetilde{R}_{d o b}{ }^{o}=0, & \widetilde{R}_{d c o}{ }^{a}=0, \\
\widetilde{R}_{d c o}{ }^{o}=0, & \widetilde{R}_{d o o}{ }^{a}=0, & \widetilde{R}_{d o o}{ }^{o}=0,
\end{array}
$$

where $R_{d c b}{ }^{a}$ denotes the components of the curvature tensor of the affine connection $\nabla$ induced in the base space $M$.

If we change horizontal planes by means of (6.5) and put

$$
\begin{aligned}
{ }^{\prime} \widetilde{R}_{d c b}{ }^{a} & =\widetilde{R}_{k j i}{ }^{h} \bar{E}^{k}{ }_{d} \bar{E}^{j}{ }_{c} \bar{E}^{i}{ }_{b} \bar{E}_{h}^{a}, \\
{ }^{a} \widetilde{R}_{d c b}{ }^{o} & =\widetilde{R}_{k j i}{ }^{h} \bar{E}^{k}{ }_{d} \bar{E}^{j}{ }_{c} \bar{E}^{i}{ }_{b} \bar{E}_{h},
\end{aligned}
$$

where $\bar{E}_{\imath}^{a}, \bar{E}_{\imath}, \bar{E}_{b}^{h}$ and $\bar{E}^{h}$ are defined by (6.6), we find

$$
{ }^{\prime} \widetilde{R}_{d c b}{ }^{a}=\widetilde{R}_{d c b}{ }^{a},
$$

$$
{ }^{\prime} \widetilde{R}_{d c b}{ }^{o}=\widetilde{R}_{d c b}{ }^{o}-\widetilde{R}_{d c b}{ }^{a} \phi_{a}
$$


as consequences of (6.6). The first equation of (6.12) shows that the projection $p \widetilde{R}$ of the curvature tensor $\tilde{R}$ of the invariant affine connection $\tilde{\nabla}$ is left invariant by the change of horizontal planes. Thus, the projection $p \widetilde{R}$ of the curvature tensor $\widetilde{R}$ of the invariant affine connection $\tilde{V}$ given in $\tilde{M}$ is determined completely by giving a fibred space $(\tilde{M}, M, \pi ; \tilde{C})$ without horizontal planes. Thus we have

Propostion 6.2. Let there be given an invariant affine connection satisfying the condition (6.1) in a fibred space $(\tilde{M}, M, \pi ; \widetilde{C})$ without horizontal planes and denote by $\widetilde{R}$ its curvature tensor. The projection $p \widetilde{R}$ in the base space $M$ is completely determined independently of the choice of horizontal planes. When $p \tilde{R}$ vanishes identically, the projection of the tensor field $\tilde{R}_{k j i}{ }^{h} E_{h}$ is in $M$ completely determined independently of the choice of horizontal planes, where $\widetilde{R}_{k j i}{ }^{h}$ denote the components of $\widetilde{R}$ and $\tilde{\eta}=E_{i} d x^{2}$ is a structure 1-form.

E. Cartan's projective connection. According to E. Cartan's theory of projective connection [1], a torsionless projective connection $\Pi$ in $M$ is determined by giving arbitrarily a pair $\left(\nabla, h_{c b}\right)$ of a torsionless affine connection $\nabla$ and a tensor field $h_{c b}$ of type $(0,2)$ in $M$, and, another such pair $\left({ }^{\prime} \nabla,{ }^{\prime} h_{c b}\right)$ determines the same projective connection $\Pi$ if and only if $\left(\nabla, h_{c b}\right)$ and $\left({ }^{\prime} \nabla,{ }^{\prime} h_{c b}\right)$ are related to each other by means of $(6.8)$ with a certain covariant vector field $\phi_{b}$. The tensor fields $\widetilde{R}_{d c b}{ }^{a}$ and $\widetilde{R}_{d c b}{ }^{o}$ given by (6.9) are called the curature tensors of the projective connection II determined by $\left(\nabla, h_{c b}\right)$. Thus we have

Proposition 6. 3. Let there be given an invariant affine connection $\tilde{\nabla}$ satisfying the condition (6.1) in a fibred space $(\tilde{M}, M, \pi ; \tilde{C})$ without horizontal planes. Then a projective connection $\Pi$ is induced in the base space $M$, where $\Pi$ is determined by a pair $\left(\nabla, h_{c b}\right)$ induced in $M$ by giving a field of horizontal planes. The projection p $\widetilde{R}$ of the curvature tensor $\widetilde{R}$ of $\tilde{\nabla}$ and the projection of the tensor field $\widetilde{R}_{k j i}^{h} E_{h}$ are the curvature tensor of the induced projective connection $\Pi$, where $\widetilde{R}_{k j i}{ }^{h}$ denote the components of $\widetilde{R}$ and $\tilde{\eta}=E_{i} d x^{2}$ is a structure 1-form.

In E. Cartan's theory [1], a projective connection is determined by giving an arbitrary pair $\left(\nabla, h_{c b}\right)$ of a torsionless affine connection $\nabla$ and a tensor field $h_{c b}$ of type (0.2) in $M$. However, in our case, the induced tensor field $h_{c b}$ satisfies necessarily the condition (2.39). That is to say, the 2 -form $\Omega=-(1 / 2)\left(h_{c b}-h_{b c}\right) d \xi^{c} \wedge d \xi^{b}$ is closed and determines the characteristic class [ $\Omega]$ of the given fibred space $(\tilde{M}, M, \pi ; \tilde{C})$ without horizontal planes.

Taking account of (6.9) and (6.10), we have

PROPOSITION 6. 4. Let there be given an invariant affine connection $\tilde{\nabla}$ satisfying the condition (6.1) in a fibred space $(\tilde{M}, M, \pi ; \widetilde{C})$ without horizontal planes. The projective connection $I I$ induced in the base space $M$ is of zero curvature (i.e., $R_{d c b}{ }^{a}=0$ and $R_{d c b^{\circ}}=0$ ) if and only of the given invariant affine connection $\tilde{\nabla}$ is of zero curvature in the total space $\tilde{M}$. 
In a fibred space $(\tilde{M}, M, \pi ; \tilde{C}, \tilde{\eta})$ with an invariant affine connection $\tilde{\nabla}$ satisfying the condition (6.1), let $\tilde{C}$ be a path in $\tilde{M}$ with respect to $\tilde{\nabla}$ and expressed by equations $x^{h}=x^{h}(t)$ in a cylindrical neighborhood $\tilde{U}, t$ being an affine parameter. Then, by means of Proposition 3.3, the projection $\mathcal{C}=\pi(\widetilde{\mathcal{C}})$ is a path in $M$ with respect to the affine connection $\nabla$ induced in $M$ and $\mathcal{C}$ has the parameter $t$ as its projective parameter. The projection $\mathcal{C}$ is expressed by equations $\xi^{a}=\xi^{a}(t)$ in $U=\pi(\tilde{U})$, the function $\xi^{a}(t)$ being defined by $\xi^{a}(t)=\xi^{a}\left(x^{h}(t)\right)$ (cf. $\S 3$ ). We define a function $\xi^{0}(t)$ along $\mathcal{C}$ by the integral

$$
\xi^{0}(t)=\int_{t_{0}}^{t} E_{i} \frac{d x^{i}}{d t} d t
$$

taken along $\mathcal{C}, t_{0}$ being a constant. Taking account of (6.3), we have along $\mathcal{C}$

$$
\frac{d \xi^{0}}{d t}=E_{i} \frac{d x^{i}}{d t}, \quad \frac{d^{2} \xi^{0}}{d t^{2}}=-h_{c b} \frac{d \xi^{c}}{d t} \frac{d \xi^{b}}{d t}-\alpha\left(\frac{d \xi^{0}}{d t}\right)^{2} .
$$

On the other hand, taking account of (3.2), (6.2) and (6.14), we find along $\mathcal{C}$

$$
\frac{d \xi^{a}}{d t}=E_{\imath}{ }^{a} \frac{d x^{i}}{d t}, \quad \frac{\delta^{2} \xi^{a}}{d t^{2}}=-2 \alpha \frac{d \xi^{0}}{d t} \frac{d \xi^{a}}{d t} .
$$

If we now define a new parameter $s$ along $C$ by the differential equation

$$
\frac{\frac{d^{2} t}{d s^{2}}}{\left(\frac{d t}{d s}\right)^{2}}=2 \alpha \frac{d \xi^{0}}{d t}
$$

then, as is well known, we have along $C$

$$
\frac{\delta^{2} \xi^{a}}{d s^{2}}=0, \quad\{t, s\}=-2 \alpha h_{c b} \frac{d \xi^{c}}{d t} \frac{d \xi^{b}}{d t}
$$

where

$$
\{t, s\}=\frac{\frac{d^{3} t}{d s^{3}}}{\frac{d t}{d s}}-\frac{3}{2}\left(\frac{\frac{d^{2} t}{d s^{2}}}{\frac{d t}{d s}}\right)^{2}
$$

is the so-called Schwarzian (cf. [1], [12], [13]). By means of the second equation of (6.17), any projective parameter $t$ of a path $\mathcal{C}$ given in $M$ is determined as a function of an affine parameter $s$ of $\mathcal{C}$ up to projective transformation $\bar{t}=(a t+b) /(c t+d)$ $(a d-b c \neq 0), a, b, c$ and $d$ being constant. 
Normal projective connection. In a fibred space $(\tilde{M}, M, \pi ; \tilde{C}, \tilde{\eta})$ admitting an invariant affine connection $\tilde{\nabla}$, which satisfies the condition (6.1), we assume now that the curvature tensor $\tilde{R}$ of $\tilde{V}$ satisfies the condition

$$
\widetilde{R}_{m j i}{ }^{m}=0,
$$

which is equivalent to the condition

$$
\widetilde{R}_{e c b}{ }^{e}=0
$$

because of (6.10). The condition (6.18) shows that the invariant affine connection $\tilde{\nabla}$ has vanishing Ricci tensor. The condition $(6.18)$ or $(6.19)$ is equivalent to the condition

$$
h_{c b}=-\frac{1}{n^{2}-1}\left(n R_{c b}+R_{b c}\right)
$$

$R_{c b}=R_{e c b}{ }^{e}$ denoting the Ricci tensor of the affine connection $\nabla$ induced in $M$. Thus, in the present case, the tensor field $h_{c b}$ is determined completely by (6. 20), if there is given a torsionless affine connection $V$ in the base space $M$. The projective connection $\stackrel{\circ}{I}$ determined in $M$ by the pair $\left(\nabla, h_{c b}\right), h_{c b}$ being given by $(6.20)$, is called the normal projective connection corresponding to the affine connection $\nabla$. Thus we have

Proposition 6. 5. Let there be given an invariant affine connection $\tilde{\nabla}$ satisfying the condition (6.1) in a fibred space $(\tilde{M}, M, \pi ; \widetilde{C})$ without horizontal planes. The projective connection induced in $M$ is the normal projective connection II corresponding to an affine connection $\nabla$, which is induced in $M$ by giving in $\tilde{M}$ a field of horizontal planes, if and only if the Ricci tensor of the connection $\tilde{\nabla}$ vanishes identically in $\tilde{M}$.

Substituting (6.20) in the first and the second equations of (6.9), we find

$$
\begin{aligned}
\widetilde{R}_{d c b}{ }^{a} & =R_{d c b}{ }^{a}+\left(\delta_{d}^{a} M_{c b}-\delta_{c}^{a} M_{d b}\right)+\left(M_{d c}-M_{c d}\right) \delta_{b}^{a}, \\
\alpha \widetilde{R}_{d c b}{ }^{o} & =\left(\nabla_{d} M_{c b}-\nabla_{c} M_{d b}\right),
\end{aligned}
$$

where the tensor field $M_{c b}$ is defined by

$$
M_{c b}=-\frac{1}{n^{2}-1}\left(n R_{c b}+R_{b c}\right) .
$$

As is well known, the right-hand sides of both equations in (6.21) are respectively the Weyl's projective curvature tensors $W_{d c b}{ }^{a}$ and $W_{d c b}$ of the connection $\nabla$, which are determined completely by the affine connection $\nabla$ induced in $M$. Thus, in this case, we have 


$$
\widetilde{R}_{d c b}{ }^{a}=W_{d c b}^{a}, \quad \widetilde{R}_{d c b}{ }^{o}=\frac{1}{\alpha} W_{d c b} .
$$

On the other hand, an affine connection $\nabla$ in $M$ is said to be projectively flat if, for any point $\mathrm{P}$ of $M$, there exists a neighborhood such that there exists in that neighborhood an affine connection which is projectively related to $\nabla$ and of zero curvature. Then, it is well known that an affine connection $\nabla$ is projectively flat, provided $\operatorname{dim} M \geqq 3$, if and only if its projective curvature tensor $W_{d c b}{ }^{a}$ vanishes identically, and, that $\nabla$ is projectively flat, provided $\operatorname{dim} M=2$, if and only if its projective curvature tensor $W_{d c b}$ vanishes identically (cf. Weyl [10]). Thus we have

PROPOSITION 6.6. Let there be given an invariant affine connection $\tilde{\nabla}$ satisfying the condition (6.1) in a fibred space $(\tilde{M}, M, \pi ; \tilde{C})$ without horizontal planes. Assume that the Ricci tensor $\widetilde{R}_{j i}=\widetilde{R}_{m j i}{ }^{m}$ vanishes identically in $\tilde{M}$. Then, the projective connection $\Pi$ induced in $M$ is of zero curvature, or equivalently, an affine connection $\nabla$ induced in $M$ by giving a field of horizontal planes is projectively flat, if and only if the given invariant affine connection $\tilde{\nabla}$ in $\tilde{M}$ is of zero curvature.

The discussion developed in this section reduces to Veblen's theory [9] if we restrict ourselves only to integrable fields of horizontal planes in fibred spaces.

\section{REFERENCES}

[1] Cartan, E., Sur les variétés à connexion projective. Bull. Soc. Math. France 52 (1924), 205-241.

[2] Dantzig, D. van, Theorie des projektiven Zusammenhangs $n$-dimensionaler Räume. Math. Ann. 106 (1932), 400-454.

[ 3 ] Hanntjes, J., On the projective geometry of paths. Proc. Edinburgh Math. Soc. 5 (1937), 103-115.

[4] Kaluza, Тн., Zum Unitätsproblem der Physik. Sitzungsber. Preuss. Akad. Wiss. (1921), 966-972.

[5] KLEIN, O., Quantentheorie und fünfdimensionaler Relativitätstheorie. Zeitschr. für Physik 37 (1926), 895-906.

[6] Mutō, Y., On some properties of a fibred Riemannian manifold. Sci. Rep. Yokohama Nat. Univ. 1 (1952), 1-14.

[7] Schouten, J. A., And J. Hanntjes, Zur allgemeinen projektiven Differentialgeometrie. Comp. Math. 3 (1936), 1-51.

[8] Thomas, T. Y., The differential invariants of generalized spaces. (1934), Cambr. Univ. Press.

[9] Veblen, O., Generalized projective geometry. J. London Math. Soc. 4 (1929), $140-160$.

[10] WeYL, H., Zur Infinitesimalgeometrie: Einordnung der projektiven und der konformen Auffassung. Göttinger Nachr. (1921), 99-112.

[11] Whitehead, J. H. C., The representation of projective spaces. Ann. Math. 32 (1936), 327-360.

[12] Y YNO, K., Les espacs à connexion projective et la géométrie projective des paths. 
Thèse, (1938), Paris.

[13] YANo, K., Projective parameters on paths in D. van Dantzig's projective space. Proc. Imp. Acad. Tokyo 20 (1944), 210-215.

[14] Yano, K., And E. T. Davies, Some local properties of fibred spaces. Kōdai Math. Sem. Rep. 11 (1959), 158-177.

[15] Yano, K., and S. Ishinara, Fibred spaces and projectable tensor fields. Perspectives in Geometry and Relativity (1966), 468-481.

[16] Yano, K., nNd M. Ohgane, On unified field theories. Ann. Math. 55 (1952) 318327.

Department of Mathematics,

Tokyo Institute of Technology. 